\title{
VLTI near-IR interferometric observations of Vega-like stars
}

\section{Radius and age of $\alpha$ PsA, $\beta$ Leo, $\beta$ Pic, $\epsilon$ Eri and $\tau$ Cet}

\author{
E. Di Folco ${ }^{1}$, F. Thévenin ${ }^{2}$, P. Kervella ${ }^{3,4}$, A. Domiciano de Souza ${ }^{5,6}$, V. Coudé du Foresto ${ }^{4}$, \\ D. Ségransan ${ }^{7}$, and P. Morel ${ }^{2}$
}

${ }^{1}$ European Southern Observatory, Karl-Schwarzschild Straße 2, 85748 Garching, Germany e-mail: edifolco@eso.org

2 Observatoire de la Côte d'Azur, BP 4229, 06304 Nice Cedex 4, France

3 European Southern Observatory, Alonso de Cordova 3107, Casilla 19001, Vitacura, Santiago 19, Chile

${ }^{4}$ Laboratoire d'Études Spatiales et d'Instrumentation Astrophysique, Observatoire de Paris, Meudon, France

5 Max-Planck-Institut für Radioastronomie, Auf dem Hügel 69, 53121 Bonn, Germany

${ }^{6}$ Laboratoire Universitaire d'Astrophysique de Nice - UNSA, CNRS UMR 6525, Parc Valrose, Nice, France

7 Observatoire de Genève, 51 chemin des maillettes, 1290 Sauverny, Switzerland

Received 3 February 2004 / Accepted 11 May 2004

\begin{abstract}
We report in this paper the direct interferometric measurement of the angular diameter of five nearby Vega-like stars: $\alpha$ PsA, $\beta$ Leo, $\beta$ Pic, $\epsilon$ Eri and $\tau$ Cet. The near-infrared ( $K$ and $H$ bands) observations were conducted at the VLTI during the commissioning period with the VINCI instrument and three different baselines ranging from $66 \mathrm{~m}$ to $140 \mathrm{~m}$. The five stellar photospheres are resolved and we derive their angular diameters with a 1 to $2 \%$ accuracy, except for $\beta$ Pic (14\%). We discuss the detectability and the influence of a possible small amount of warm circumstellar dust on our measurements. In addition, we have used the stellar evolution code CESAM (Morel 1997) to compare the computed fundamental parameters to the observed values (linear diameter, luminosity, temperature and chemical abundance). As a result of the simulation, the age of the stars is inferred and found to be in good agreement with previous estimates from various other methods.
\end{abstract}

Key words. techniques: interferometric - infrared: stars - stars: fundamental parameters - stars: circumstellar matter stars: general

\section{Introduction}

During its first two years of operation, the VLT Interferometer has demonstrated its ability to determine very accurate fundamental parameters for the brightest southern stars. Precise stellar diameters and masses and even detection of rotation induced asymmetries or limb-darkening effects were made possible thanks to accurate measurements of the visibility amplitude. Together with asteroseismic observations, such direct measurements of size of photospheres will help to constrain the models of atmosphere and internal structure and to determine careful estimations of stellar masses and ages. In the present paper, we further explore main sequence (MS) stars and focus on the study of bright and nearby MS dwarf stars associated with debris-disks: the prototypic stars $\alpha$ PsA (HD 216956), $\beta$ Pic (HD 39060) and $\epsilon$ Eri (HD 22049), as well as $\beta$ Leo (HD 102647) and $\tau$ Cet (HD 10700).

Vega/ $\beta$ Pic-type stars are MS or "old Pre-MS" stars of spectral type A to $\mathrm{M}$ presenting a far infrared (IR) excess.
Discovered with the IRAS satellite (Aumann et al. 1984), this excess has been commonly identified with second-generation circumstellar (CS) dust in optically thin disks, presumably cleared of gas and possibly associated with planetary systems. Submillimetric interferometers have resolved a few disks through the thermal emission of cool grains (e.g., Greaves et al. 1998; Holland et al. 1998), revealing extended structures analog to the Kuiper Belt in our solar system. For the closest Vega-like stars, adaptive optics and coronagraphs in the visible and IR have provided high resolution images of scattered and thermal light down to 15 astronomical units (AU) from the central stars, thus unveiling asymmetries (like disk warps around $\beta$ Pic or asymmetric rings) interpreted as the suspected signature of already formed planets (Mouillet et al. 1997; Artymowicz et al. 1997). Sub-mm images have also revealed large scale asymmetries and clumpy structures around $\epsilon$ Eridani (Greaves et al. 1998) and more recently around Fomalhaut (Holland et al. 2003) which were tentatively interpreted as the interaction of a planet with the 
Table 1. Observational parameters for the selected targets.

\begin{tabular}{|c|c|c|c|c|c|c|c|c|c|}
\hline Star & Spec. type & $\begin{array}{c}\text { Distance }^{a} \\
\text { pc }\end{array}$ & $m_{V}$ & $m_{H}$ & $m_{K}$ & $\begin{array}{c}v_{\mathrm{eq}} \sin i^{\mathrm{b}} \\
\mathrm{km} \mathrm{s}^{-1}\end{array}$ & $L_{\mathrm{IR}} / L_{\star}$ & $\begin{array}{c}\mathrm{FOV}^{c} \\
\mathrm{UTs}\end{array}$ & $\begin{array}{l}\mathrm{FOV}^{c} \\
\text { Sids }\end{array}$ \\
\hline$\tau$ Cet (HD 10700) & G8V & $3.65 \pm 0.01$ & 3.49 & 1.73 & 1.66 & 0.4 & $1.2 \times 10^{-5}$ & & $5-10 \mathrm{AU}$ \\
\hline$\epsilon$ Eri (HD 22049) & $\mathrm{K} 2 \mathrm{~V}$ & $3.22 \pm 0.01$ & 3.73 & 1.79 & 1.69 & 1.7 & $8 \times 10^{-5}$ & $0.2 \mathrm{AU}$ & 4.5-9 AU \\
\hline$\beta$ Pic (HD 39060) & A5V & $19.28 \pm 0.19$ & 3.86 & 3.50 & 3.47 & 130 & $3 \times 10^{-3}$ & $1.3 \mathrm{AU}$ & \\
\hline$\beta$ Leo (HD 102647) & $\mathrm{A} 3 \mathrm{~V}$ & $11.10 \pm 0.11$ & 2.14 & 1.94 & 1.99 & 128 & $1.9 \times 10^{-5}$ & & $15-30 \mathrm{AU}$ \\
\hline$\alpha$ PsA (HD 216956) & A3 $\mathrm{V}$ & $7.69 \pm 0.05$ & 1.10 & 1.05 & 0.99 & 93 & $4.6 \times 10^{-5}$ & $0.5 \mathrm{AU}$ & $10-20 \mathrm{AU}$ \\
\hline
\end{tabular}

${ }^{a}$ From Hipparcos, (Perryman et al. 1997).

${ }^{b}$ From Royer et al. (2002) and Saar et al. (1997).

${ }^{c}$ Radius of the single-mode ( $K$ band) field of view (FOV) with $8 \mathrm{~m}$ UTs and $40 \mathrm{~cm}$ siderostats (elliptical projected pupil).

external part of the disk (e.g., Quillen \& Thorndike 2002; Wyatt $\&$ Dent 2002).

However, current techniques do not provide yet enough spatial resolution and dynamic range to probe the first $10 \mathrm{AU}$ where planets or planetesimals might be found. A $10 \mathrm{~m}$ class telescope can reach a theoretical angular resolution of about $200 \mathrm{mas}$ at $10 \mu \mathrm{m}(20 \mathrm{AU}$ at a distance of $100 \mathrm{pc})$, and longbaseline optical interferometers are required in order to probe the innermost regions. Only mid-IR imaging has succeeded so far in revealing dust grains as close as $10 \mathrm{AU}$ from the central star for the nearby $\beta$ Pic, owing to the favorable dust/stellar brightness ratio in this spectral domain (Pantin et al. 1997). With baseline lengths in the order of $100 \mathrm{~m}$, the VLT interferometer (Glindemann et al. 2000) can reach a resolution of about 4 mas (less than $0.1 \mathrm{AU}$ at the distance of $\beta$ Pic) in a 70 mas field of view (FOV) with $8 \mathrm{~m}$ telescopes operating at $\lambda=2.2 \mu \mathrm{m}$. Thus, the single-mode FOV of the telescope (Table 1) makes it possible to concentrate the observations on the central few AUs.

The simplest result that can be derived from interferometric observations is the size of the stellar photospheres, obtained by adjusting the visibility curve of a disk to the data. From the stellar size one can infer an improved estimation of the age. Precise age determination should feed the debate on the relationship between the fractional dust luminosity and the age of Vega-like stars (see recent discussion by Decin et al. 2003).

IR interferometry can also potentially reveal the presence of very small amounts of dust, whose signature in the visibility function is proportional to the dust to star flux ratio. Observing at $2 \mu \mathrm{m}$ could reveal dust grains close to the sublimation limit ( $T \lesssim 1500 \mathrm{~K}$ for refractory material), although from theoretical modeling (Dent et al. 2000) only a very small amount of dust is expected to be found in the inner region of these disks. $K$-band excesses have never been reported so far around MS stars older than $\sim 10 \mathrm{Myr}$, but the combined photometric and modeling accuracy generally does not exceed a few percent.

We have used the VLTI first to constrain the photospheric parameters of five bright debris disk stars located within $20 \mathrm{pc}$ from the Sun. With ages from $10 \mathrm{Myr}$ to about $7.5 \mathrm{Gyr}$, they cover a wide range of representative cases of Vegalike stars. They were selected on brightness criteria since the sensitivity of VINCI is limited to $K \sim 3$ with the $40 \mathrm{~cm}$ siderostats. The derived angular and linear diameters are presented in Sect. 3. Based on a basic modeling of a dusty ring, we have derived from the observed visibility variations an upper limit on the presence of possible CS material (Sect. 4). Finally, we model the central stars (Sect. 5) with the stellar evolution code CESAM (Morel 1997), using the observable constraints and the newly derived diameters in order to estimate the age of the program stars.

\section{Interferometry at the VLTI with VINCI}

\subsection{Instrumental configuration}

Our observations were conducted at the VLTI (Fig. 1) operated by the European Southern Observatory on top of Cerro Paranal (Chile). In its early phase of commissioning, the interferometer could be used in a limited number of configurations (Fig. 1): occasionally two of the four $8 \mathrm{~m}$ Unit Telescops (namely UT1 and UT3, on a $102.5 \mathrm{~m}$ long baseline) were available but most of observations were performed with the two $40 \mathrm{~cm}$ test siderostats on the $66 \mathrm{~m} \mathrm{E0-G1} \mathrm{and} 140 \mathrm{~m} \mathrm{B3-M0}$ baselines, which have almost perpendicular orientations (ensuring a favorable configuration for the detection of any asymmetry in the source brightness profile). The collected beams were then combined coherently with VINCI, the VLT INterferometer Commissioning Instrument (Kervella et al. 2000; 2002).

VINCI uses a fiber-based two-way beam combiner which filters out the atmospheric turbulence and converts it into temporal photometric fluctuations (except the piston mode). The scans are obtained by a temporal modulation of the optical path. In its standard mode, it has the spectral range and resolution of a standard $K$ broadband filter $(\lambda=2.0-2.4 \mu \mathrm{m})$, VINCI was also occasionally operated in the $H$ band $(\lambda=1.5-1.8 \mu \mathrm{m})$, replacing the fibers by an integrated optics beam combiner, IONIC (Laurent et al. 2002). The processing is based on a wavelet analysis of the fringe pattern. Details can be found in Kervella et al. (2004a). The resulting coherence factors measure the amplitude of the complex degree of coherence of the two beams at the spatial frequency determined by the baseline and effective wavelength. 


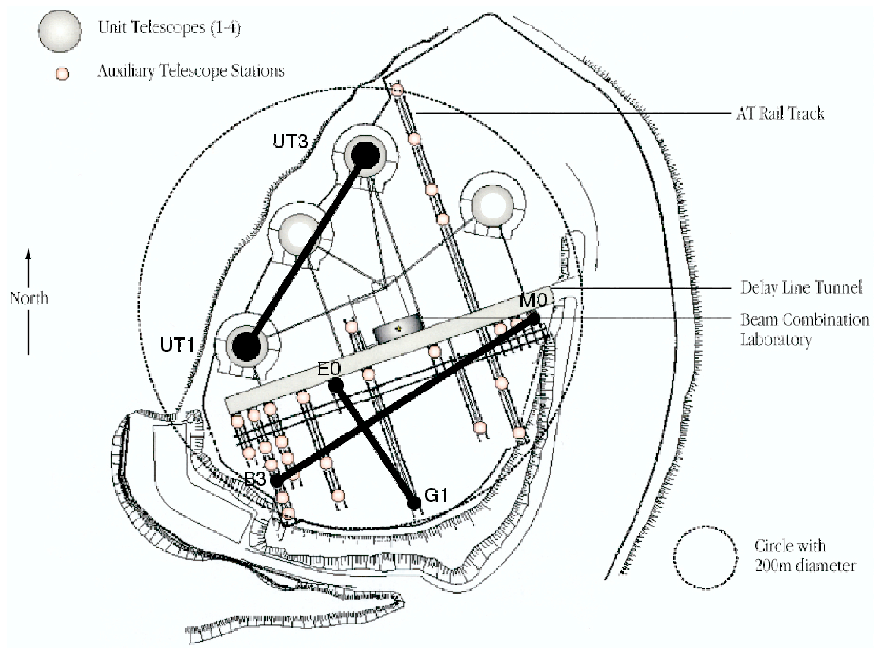

Fig. 1. Geographic overview of the VLTI telescope stations on top of Cerro Paranal. Highlighted are the three baselines which have been used for the reported observations.

\subsection{Effective wavelength}

The effective wavelength of each measurement differs slightly from the central wavelength of the broad-band filter used. The total spectral transmission has to be taken into account as well as the spectrum of the target itself. The global VLTI transmission was modeled (including the atmosphere) and the instrumental transmission was measured on a series of bright stars of various spectral types (see Kervella et al. 2003, for details). The adopted values are summarized in Table 1 . They were used to fit the monochromatic uniform angular diameters presented in Sect. 3, which are an excellent approximation of the broadband diameters until squared visibilities are above $\sim 20 \%$. This bandwidth smearing effect was taken into account in the calibration of $\tau$ Ceti only, since one of its calibrators $(\theta$ Cet) is significantly resolved $\left(V^{2} \sim 15 \%\right)$ on the $140 \mathrm{~m}$ long baseline.

\subsection{Calibration: From coherence factors to visibilities}

The calibration is performed by converting the raw squared coherence factors $\left(\mu^{2}\right)$ into normalized visibilities. The transfer function is estimated with reference to interleaved observations of stars with known diameter. Most of our calibrators were chosen from the catalog by Bordé et al. (2002), based on the spectrophotometric work of Cohen et al. (1999). Calibrator sizes are known with a typical precision of 1 to $2 \%$ (see Table 2).

To compute the visibility uncertainty, we have separated the statistical error (related to the dispersion of $\mu^{2}$ values) and the systematic error (induced by the uncertainty on the calibrator's diameter). Angular diameter values are then presented in the form $\theta \pm \sigma_{\text {stat }} \pm \sigma_{\text {syst }}$. Systematic errors dominate all the angular diameter uncertainties. To reduce the systematics, we used as many calibrators as possible. For most of our visibility measurements, we have actually computed independent angular diameters for each calibrator and averaged the individual values to infer the adopted final diameter. The systematic errors thus decrease with the number and quality of the available calibrators.

\section{Observations and diameter determination}

\subsection{Interferometric data acquisition and calibration}

The data reported in this paper were collected between November 2001 and May 2003. Neither an adaptive optics system nor a fringe tracker was used to compensate for the atmospheric corrugation of the wavefronts and jitter of the fringe pattern. Only a tip-tilt correction at the Coudé focus was applied during the UTs observations of $\alpha$ PsA.

Each visibility measurement is derived from a typical 500 scan observation set (equivalent to 6 min of effective observing time for one visibility point without calibration), each scan corresponding to a temporal path-length modulation of about $150 \mu \mathrm{m}$. The resulting fringe frequency is between $500-700 \mathrm{~Hz}$ with the UTs and $300 \mathrm{~Hz}$ with the siderostats, depending on the adopted integration time.

The list of calibrating stars can be found in Table 2 with their estimated angular diameters. The large number of calibrators observed during these two years allowed us to evidence some discrepancies for a few targets between the diameter predicted by Cohen et al. (1999) and that derived from the observed visibilities. Using other reference stars from Cohen's catalog, we have determined new diameters for five calibrators, which showed clear departures from their expected visibilities: $\delta$ Eri, 39 Eri and $\lambda$ Gru, 16 Vir and $\theta$ Cet. The revised values are also reported in Table 2, they ensure a better consistency between visibilities of all observed reference stars from Cohen's catalog.

\subsection{Visibilities and angular diameters}

In the following sections, the measured visibilities will be used to fit models representative of the stellar surface brightness. The uniform disk (UD) model assumes a constant intensity, whereas the limb darkened (LD) model is characterized by a decreasing intensity toward the edge of the stellar disk, that is more representative of the actual surface brightness. We have used the UD model to fit the interferometric data. The UD angular diameters are then converted into LD diameters, as explained in Sect. 3.3, before computing the linear stellar radii.

\subsection{1. $\tau$ Ceti (HD 10700)}

$\tau$ Ceti is an old metal deficient star of the galactic disk located $3.6 \mathrm{pc}$ away from the Sun. Its weak fractional dust luminosity was estimated by Backman et al. (1986) to be $f_{\mathrm{d}}=1.2 \times 10^{-5}$. The CS emission has been very recently resolved by Greaves et al. (2004), who found a central radius at $60 \mathrm{AU}$ for the dust from sub-mm observations with SCUBA. $\tau$ Ceti is also a prime target for the asteroseismic campaigns. Its size has already been inferred from the VLTI data by Pijpers et al. (2003) and we will give in this section a revised value for its angular diameter, on the basis of a more extended calibration procedure and after the revision of its main calibrator's size, $\theta$ Cet. Between 
Table 2. Relevant parameters for the calibrators used.

\begin{tabular}{|c|c|c|c|c|c|c|c|c|c|}
\hline Name & HD number & Sp. type & $m_{\mathrm{V}}$ & $m_{\mathrm{K}}$ & $T_{\mathrm{eff}^{a}}^{a}$ & $\log g^{a}$ & $\theta_{\mathrm{LD}} \pm \sigma$ mas & $\theta_{\mathrm{LD}}^{\text {revised }} \pm \sigma$ mas $^{b}$ & Calibrated target(s) \\
\hline$\delta$ Psc & HD 4656 & K4IIIlb & 4.44 & 0.83 & 3920 & 1.7 & $3.80 \pm 0.10$ & & $\alpha$ PsA \\
\hline$\theta$ Cet & HD 8512 & K0IIb & 3.60 & 1.48 & 4720 & 2.6 & $2.76 \pm 0.03$ & $2.84 \pm 0.03$ & $\tau$ Cet \\
\hline$\delta$ Phe & HD 9362 & K0IIb & 3.95 & 1.61 & 4720 & 2.6 & $2.24 \pm 0.02$ & & $\epsilon$ Eri \\
\hline$\chi$ Phe & HD 12524 & K5III & 5.16 & 1.29 & 3780 & 1.9 & $2.77 \pm 0.03$ & & $\beta$ Pic \\
\hline$\delta$ Eri & HD 23249 & K0IV & 3.51 & 1.40 & 4720 & 2.6 & $2.48 \pm 0.03$ & $2.37 \pm 0.03$ & $\epsilon$ Eri \\
\hline 39 Eri & HD 26846 & K3III & 4.90 & 2.25 & 4220 & 2.2 & $1.86 \pm 0.02$ & $1.79 \pm 0.03$ & $\epsilon$ Eri \\
\hline$\epsilon$ Ori & HD 37128 & B0Ia & 1.70 & 2.18 & 30000 & 3.6 & $0.69 \pm 0.04$ & & $\tau$ Cet \\
\hline$\gamma^{2} \mathrm{Vol}$ & HD 55865 & KOIII & 3.77 & 1.52 & 4720 & 2.6 & $2.50 \pm 0.06$ & & $\beta \mathrm{Pic}$ \\
\hline 6 Leo & HD 82381 & $\mathrm{~K} 2.5 \mathrm{IIIb}$ & 5.07 & 1.90 & 4335 & 2.3 & $2.16 \pm 0.03$ & & $\beta$ Leo \\
\hline $16 \mathrm{Vir}$ & HD 107328 & KOIII & 5.00 & 2.20 & 4350 & 2.3 & $1.75 \pm 0.02$ & $1.87 \pm 0.03$ & $\beta$ Leo \\
\hline$\omega$ Cen & HD 110458 & K0III & 4.66 & 2.30 & 4720 & 2.6 & $1.70 \pm 0.02$ & & $\beta$ Leo \\
\hline $70 \mathrm{Aql}$ & HD 196321 & K5II & 4.90 & 1.21 & 3780 & 1.9 & $3.27 \pm 0.04$ & & $\alpha$ PsA \\
\hline $7 \mathrm{Aqr}$ & HD 199345 & K5III & 5.50 & 2.01 & 3780 & 1.9 & $2.14 \pm 0.02$ & & $\alpha$ PsA \\
\hline$\lambda \mathrm{Gru}$ & HD 209688 & K3III & 4.48 & 1.68 & 4210 & 2.2 & $2.71 \pm 0.03$ & $2.75 \pm 0.03$ & $\alpha$ PsA \\
\hline HR 8685 & HD 216149 & M0III & 5.41 & 1.60 & 3660 & 1.4 & $2.07 \pm 0.02$ & & $\alpha$ PsA \\
\hline$\psi^{1}$ Aqr & HD 219449 & K1III & 4.21 & 1.76 & 4580 & 2.5 & $2.24 \pm 0.03$ & & $\alpha$ PsA \\
\hline
\end{tabular}

a From Cayrel de Strobel et al. (1992).

${ }^{b}$ Angular diameters from this work, limb darkened (LD) diameters were obtained with Claret (2000) coefficients using the Handbury-Brown et al. (1974) approximation (see Sect. 3.3 for details).



Fig. 2. Summary of the visibility measurements on $\tau$ Cet with the E0-G1 and B3-M0 siderostats baselines. The UD model fit leads to $\theta_{\mathrm{UD}}=2.032 \pm 0.014 \pm 0.028$ mas.

September and November 2002, more than 7500 scans $(\sim 1.5 \mathrm{~h})$ were acquired on this star, $90 \%$ of which were obtained on the $140 \mathrm{~m} \mathrm{~B} 3-\mathrm{M} 0$ baseline. The associated calibrators are $\theta$ Cet, $\epsilon$ Ori, HR 8685, $\delta$ Phe and $\alpha$ PsA (measured hereafter in Sect. 3.2.5). The measurements on the $66 \mathrm{~m}$ and $140 \mathrm{~m}$ long

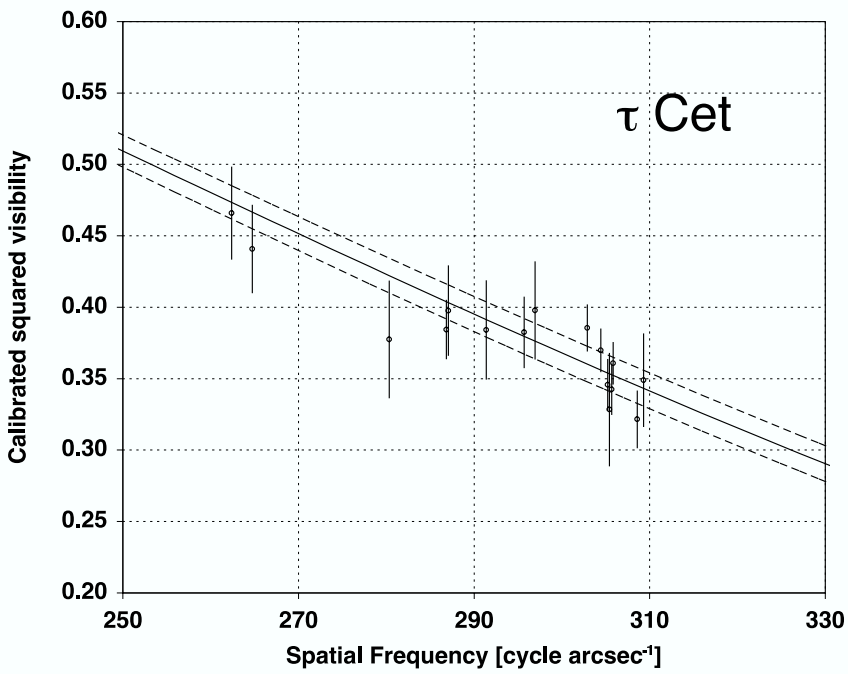

Fig. 3. Enlargement of Fig. 2 showing the high spatial frequency points obtained with the B3-M0 $140 \mathrm{~m}$ baseline (siderostats).

baselines are presented in Figs. 2 and 3. The statistical uncertainty is significantly larger on the short baseline, presumably due to stronger atmosphere induced instabilities (piston). The low number of observation sets on the short baseline does not allow us to investigate possible discrepancies between 
both baselines. The few visibilities obtained on E0-G1 are nevertheless consistent within their error bars with the equivalent uniform disk diameter derived from the $140 \mathrm{~m}$ baseline data: $\theta_{\mathrm{UD}}=2.032 \pm 0.014 \pm 0.028$ mas. The first error refers to the statistical dispersion of the $V^{2}$ measurements whereas the second corresponds to the resulting systematics due to the uncertainty on the calibrators diameters. It results in a final error of 0.031 mas (1.5\%). Saar et al. (1997) report a low rotational velocity $v \sin i=0.4 \pm 0.4 \mathrm{~km} \mathrm{~s}^{-1}$, consistent with a nearly poleon view and implying no azimuth angle dependence of the visibility. As can be seen in Fig. 3, the squared visibilities never drop below 0.3 and we could neglect (as for most other stars) the wide band chromatic effect (bandwidth smearing).

\subsection{2. $\epsilon$ Eridani (HD 22049)}

The nearby ( $3.2 \mathrm{pc}$ ) sun-like K2 dwarf star $\epsilon$ Eri is a chromospherically active star. It has been found to be orbited by a dusty ring of cold grains resolved at sub-mm wavelengths (Greaves et al. 1998). The ring is seen nearly face-on and its fractional luminosity is estimated to be $f_{\mathrm{d}}=8 \times 10^{-5}$. Clumps have been evidenced by Holland et al. (2003) and are thought to originate from the interaction of the disk with a massive planet. The star has been observed with the same baselines as $\tau$ Cet and in addition with the UT1-UT3 $102.5 \mathrm{~m}$ long baseline (see Fig. 1 for the orientations).

About 8800 scans (equivalent to $\sim 2 \mathrm{~h}$ of integration) were successfully processed, the UTs data representing $30 \%$ of the total scans. The two major reference stars chosen for the data calibration are 39Eri (for the UTs data) and $\delta$ Eri (for the siderostats observations). Although both calibrators are from the Cohen catalog, their size has been revised thanks to additional VLTI observations and the adopted diameters are presented in Table 2. The resulting visibilities plotted in Fig. 4 are very consistent for all baselines and lead to similar diameter estimations. The fitted UD diameter is $\theta_{\mathrm{UD}}=2.093 \pm 0.010 \pm$ 0.027 mas. With a global uncertainty of 0.029 mas, the relative error on its angular diameter is $1.4 \%$. This result is the first direct measurement of this star.

No significant azimuthal dependence of the visibility was observed at our level of precision. Rotational velocity measurements are consistent with an almost pole-on view (Saar et al. 1997). The inclination of the disk on the sky plane was estimated to be close to $20 \mathrm{deg}$ (Greaves et al. 1998) and it is thought to be aligned with the equatorial plane of the star. Our measurement is in agreement with this configuration.

\subsection{3. $\beta$ Leonis (HD 102647)}

$\beta$ Leo is a relatively young A 3 dwarf star located $11 \mathrm{pc}$ away from the Sun. Its fractional IR luminosity was first established by Aumann (1985) to be $f_{\mathrm{d}}=1.9 \times 10^{-5}$, Laureijs et al. (2002) measured the $25 \mu \mathrm{m}$ emission with ISO and derived from the $25 / 60 \mu \mathrm{m}$ flux ratio a dust temperature of $83 \pm 5 \mathrm{~K}$. The disk has not been imaged yet.

A few sets of observations were obtained with VINCI on the $140 \mathrm{~m}$ long siderostat baseline (B3-M0) in April-May 2003.

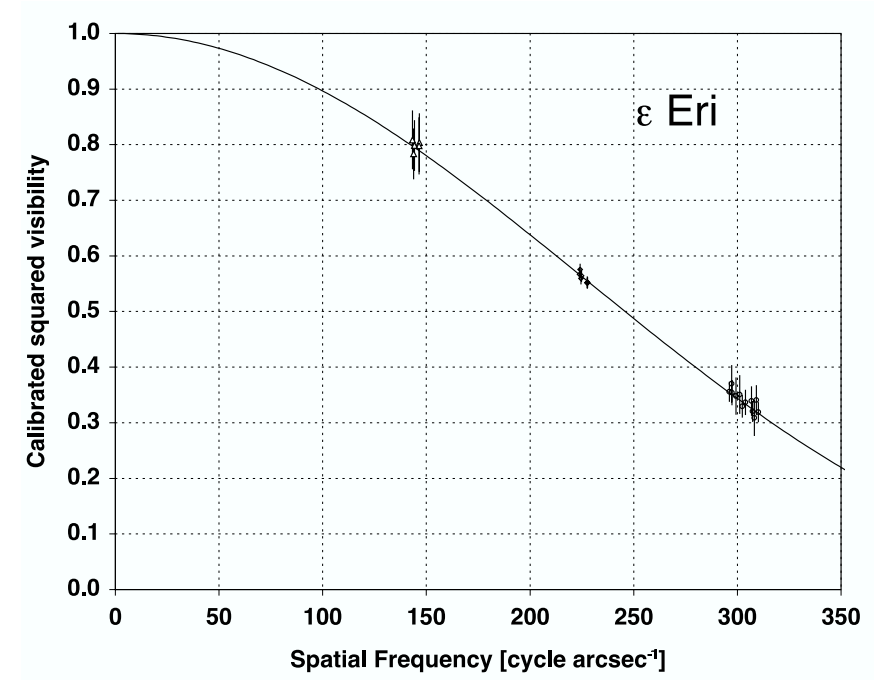

Fig. 4. Summary of the visibility measurements on $\epsilon$ Eri with the three baselines in the $K$ band and the associated best UD model fit: $\theta_{\mathrm{UD}}=$ $2.093 \pm 0.010 \pm 0.027$ mas.

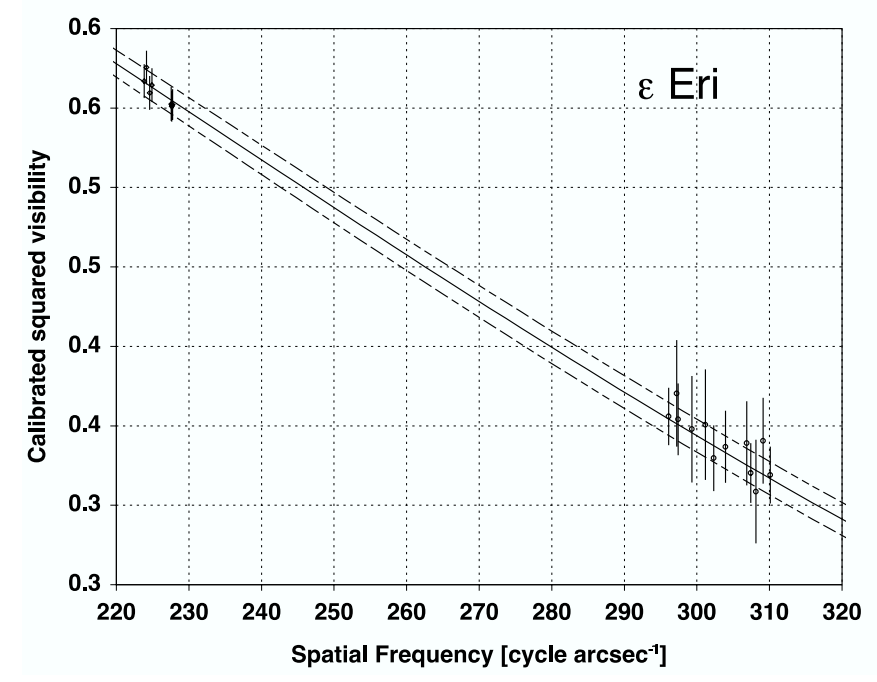

Fig. 5. Enlargement of Fig. 4 presenting the best UD model fit with its total (statistic and systematic) uncertainty in the high spatial frequency domain.

A total of 3300 scans were selected and processed (about $45 \mathrm{~min}$ of integration). The angular size of the main calibrator (16 Vir) was also revised since a discrepancy of $\sim 3 \sigma$ from Cohen's prediction was evidenced by other long baseline observations (see Table 2). Two additional calibrators, namely 6 Leo and HR 4831, were also occasionally used. The squared visibilities presented in Fig. 6 show some dispersion and the fit of the UD model leads to $\theta_{\mathrm{UD}}=1.429 \pm 0.014 \pm 0.023$ mas (relative error $1.9 \%$ ). Due to the small number of observations, the reduced chi-square is $\chi_{\text {red }}^{2}=1.3$, but the goodness of the fit $(23 \%)$ is still reasonable. 


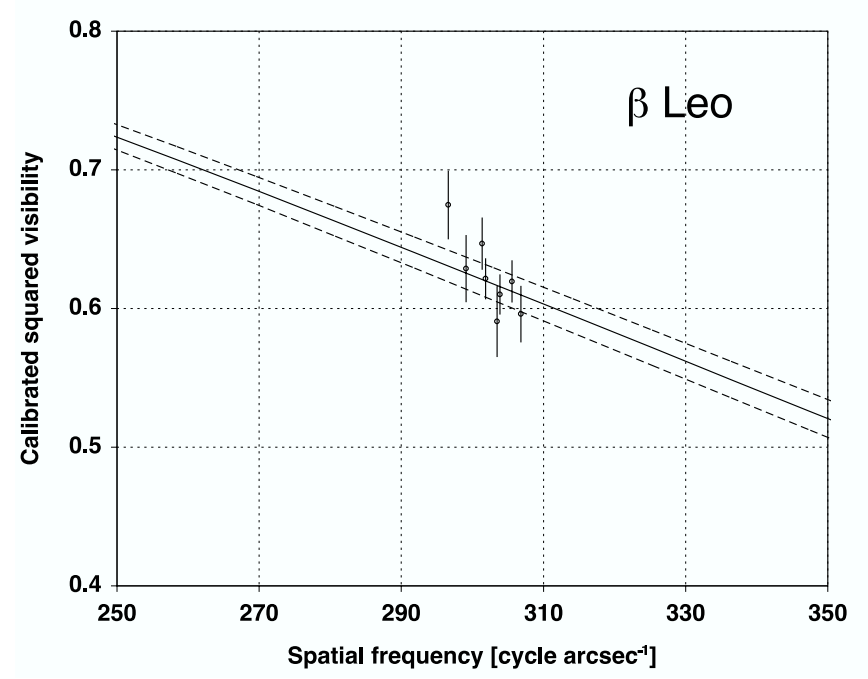

Fig. 6. Overview of the visibilities measured on $\beta$ Leo with the B3-M0 $140 \mathrm{~m}$ long baseline in Spring 2003 and best UD model fit with its $1 \sigma$ (total) error: $\theta_{\mathrm{UD}}=1.429 \pm 0.014 \pm 0.023$ mas.



Fig. 7. Measured visibility amplitude for $\beta$ Pic with the UT1-UT3 $102.5 \mathrm{~m}$ baseline in November 2001 and best UD model fit (solid) with its $1 \sigma$ error (dashed): $\theta_{\mathrm{UD}}=0.84 \pm 0.06 \pm 0.10$ mas.

\subsection{4. $\beta$ Pictoris (HD 38060)}

Most studied among the Vega-like prototype stars, $\beta$ Pictoris was observed only once in November 2001 with UT1 and UT3 (102.5 m long baseline). With a $K$ magnitude of 3.47 (19.4 pc away), it could not be observed with the siderostats. It has the largest IR excess observed so far with a fractional luminosity $f_{\mathrm{d}}=3 \times 10^{-3}$. Its edge-on disk has been resolved at optical and IR wavelengths. Warps and a tilted inner disk were evidenced and attributed to the interaction of the dust with a possible giant planet. The UT1-UT3 baseline orientation is almost colinear to the major axis of the circumstellar disk projected on the sky.

Only five observation sets were obtained for a total of $\sim 1650$ processed scans at two different acquisition frequencies. $\chi$ Phe and $\gamma^{2}$ Vol were used as calibrators. The major result, as shown in Fig. 7 , is that $\beta$ Pic is marginally resolved for the first time with an average squared visibility of $92.5 \%$.

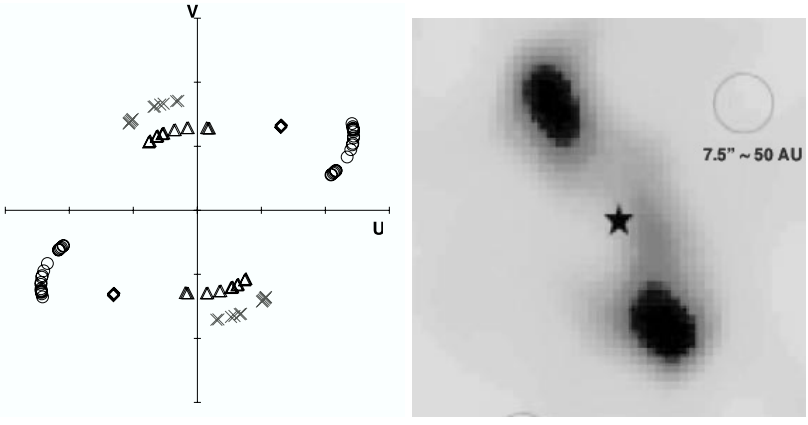

Fig. 8. UV plane coverage (North is up and East to the right) for $\alpha$ PsA observations and comparison with the orientation of the disk resolved in the sub-mm (adapted from Holland et al. 2003). Its major axis is colinear to the short E0-G1 baseline (triangles for the $K$ band, crosses for the $H$ band) and perpendicular to UT1-UT3 (diamonds) and B3-M0 (circles) . The region probed by our interferometric observations is smaller than the SCUBA beam at $450 \mu \mathrm{m}$.

The low number of observation sets recorded does not provide yet a very accurate estimation of the equivalent UD diameter: $\theta_{\mathrm{UD}}=0.84 \pm 0.06 \pm 0.10$ mas. The large total relative error (about 14\%) is due to unfavorable atmospheric conditions (which can account for instability of the TF, bad flux injection conditions and strong differential piston), and tends to increase the dispersion of the visibilities within an observation set. The small number of calibrators available explain the large systematic (external) uncertainty.

\subsection{5. $\alpha$ Piscinis Austrini (HD 216956)}

Fomalhaut ( $\alpha$ PsA) is one of the 20 brightest stars in the sky. The disk around this A3V star (at $7.7 \mathrm{pc}$ ) has been imaged at 850 and $450 \mu \mathrm{m}$ (Holland et al. 1998, 2003). A dust ring of cold grains is seen $\sim 20 \mathrm{deg}$ from edge-on, with a large radius of about 135 AU. A ring asymmetry has been evidenced, accounting for some $5 \%$ of the dust cloud flux and could be attributed to collisions or resonant trapping by a planet orbiting the star (Wyatt et al. 2002; Kuchner et al. 2003). We have observed Fomalhaut between November 2001 and October 2002 with three baselines of various length and orientation: E0-G1 (66 m) and B3-M0 $(140 \mathrm{~m})$ with the siderostats, and UT1-UT3 in November 2001 shortly after the first fringes with $8 \mathrm{~m}$ telescopes (see Fig. 8). More than 13000 scans $(\sim 2.6 \mathrm{~h})$ were recorded in total, $50 \%$ on the long baseline B3-M0, 35\% with E0-G1 and 15\% with UT1-UT3. In addition, Fomalhaut was observed during technical measurements in the $H$ band with the IONIC integrated optics beam combiner in July 2002 (Kern et al. 2002). We were able to take advantage of some of these technical observations when suitable calibrators were interleaved during the night. A total of 1600 scans passed successfully the selection process and are presented below. The accuracy of these $H$ band measurements is however lower than in the $K$ band since the beam combiner comprises only one interferometric signal (instead of two phase-opposite interferometric outputs in the classical $K$ band coupler), and the atmospheric fluctuations are faster at shorter wavelengths. 


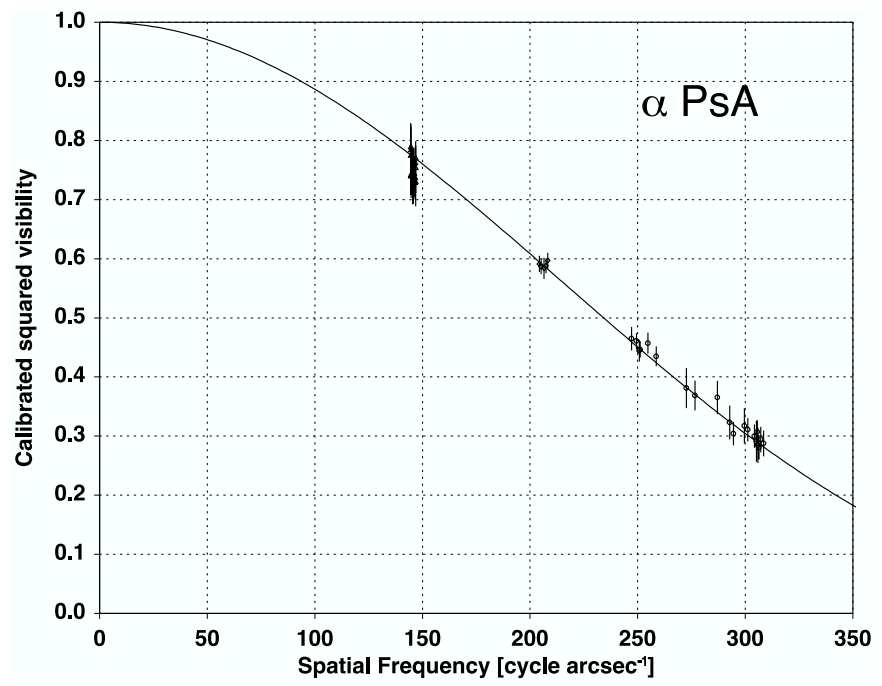

Fig. 9. Overview of the azimuth averaged $K$ band visibilities obtained on three baselines for $\alpha$ PsA. The best UD model fit is superimposed (solid line): $\theta_{\mathrm{UD}}=2.197 \pm 0.011 \pm 0.020$ mas. The low spatial frequency measurements do not contribute much to the global fit due to their larger uncertainty.

The UV plane coverage, presented in Fig. 8, illustrates the super-synthesis effect (due to Earth rotation), which allows to cover a range of azimuth angles with a single baseline. The map of the star with its extended CS dust disk (as detected at sub-mm wavelengths) is presented in order to compare the orientation of the baselines with the circumstellar environment. The E0-G1 observations correspond to the measurement of the intensity projected along the major-axis of the extended dust disk. If we assume that this disk is weakly inclined on the equatorial plane of the star, we will measure with this baseline intensity profiles oriented along (or close to) the equatorial diameter. Conversely, the B3-M0 $140 \mathrm{~m}$ baseline provides an estimation of intensity profiles along the polar direction of the star, perpendicularly to the ring major axis. It should be emphasized again that the interferometric FOV is much narrower than the structures revealed by the sub-mm observations (actually smaller than SCUBA beam size at $450 \mu \mathrm{m}$ ). We are thus probing only the very inner part of the stellar environment (over a diameter of $1 \mathrm{AU}$ with the UTs and 20 to $40 \mathrm{AU}$ with the siderostats, at the distance of Fomalhaut, see Table 1).

The result of the $K$ band observations is shown in Figs. 9 and 10 . Three different calibrators ( $\lambda$ Gru, HR 8685 and 7 Aqr) were used on the $140 \mathrm{~m}$ baseline, thus reducing the systematic errors. The UTs measurements were calibrated with HR 8685 alone whereas the EO-G1 observations were mainly calibrated with $\lambda$ Gru (and marginally with $70 \mathrm{Aql}, \psi^{1}$ Aqr and $\delta$ Psc). The visibilities measured on the long UTs and siderostats baseline are very consistent and lead to the angular diameters $\theta_{\mathrm{UD}}=2.192 \pm 0.021 \pm 0.015 \mathrm{mas}(1.2 \%$ relative error $)$ and $\theta_{\mathrm{UD}}=2.191 \pm 0.013 \pm 0.019$ mas respectively $(1 \%)$. The $66 \mathrm{~m}$ observations alone lead to a slightly different diameter but with a larger error due to the relatively unstable transfer function and
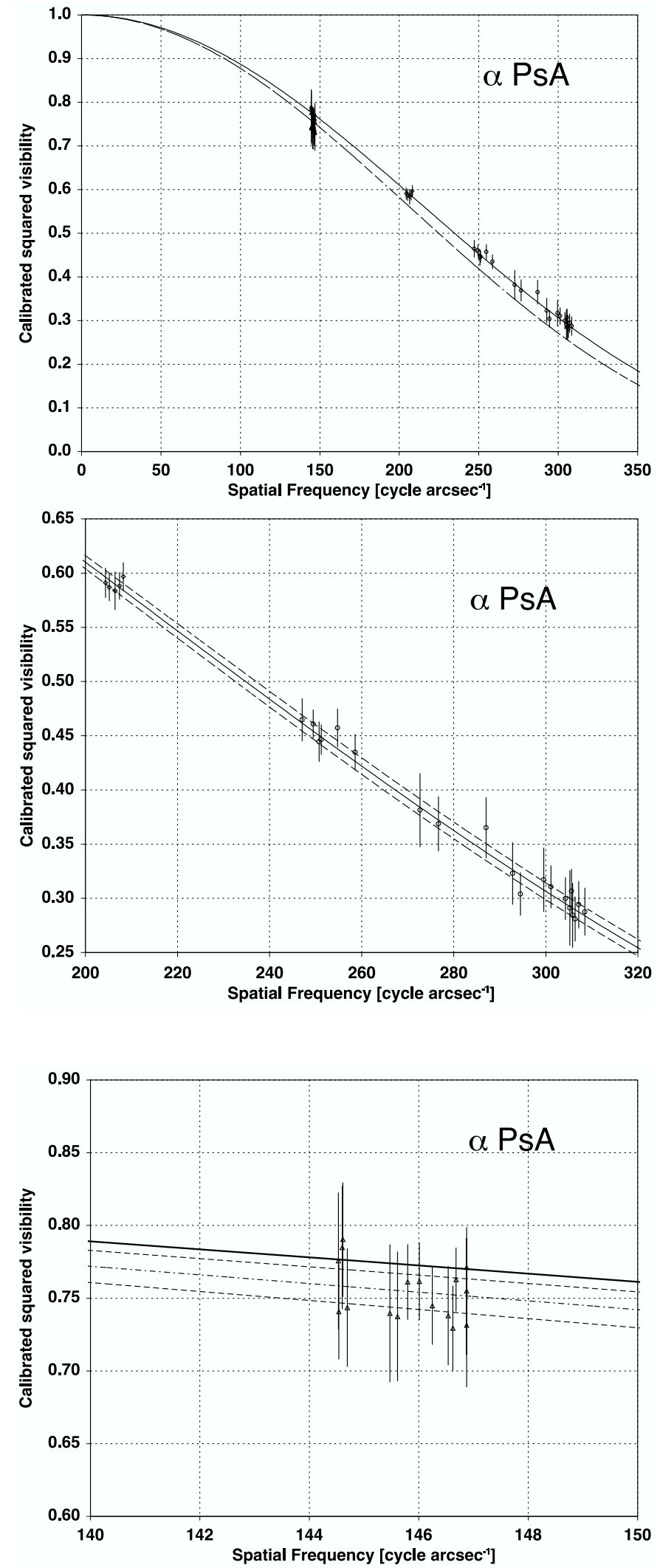

Fig. 10. Same data as in Fig. 9, but assumed to be representative of a non axisymetric brightness distribution. Best UD model fits for the perpendicular measurements are compared in the upper panel: $\theta_{\mathrm{UD}}=$ $2.191 \pm 0.011 \pm 0.018$ mas (solid) and $\theta_{\mathrm{UD}}=2.289 \pm 0.042 \pm 0.046 \mathrm{mas}$ (dash-dotted). Enlargement of the fits and related data are shown in the middle and lower panels, with their associated error (dashed). 
Table 3. Limb darkened angular diameters and linear radii for the five program stars.

\begin{tabular}{ccccccc}
\hline \hline Star & Baseline & Band $\left(\lambda_{\text {eff }}\right)$ & $\rho^{a}$ & $\theta_{\mathrm{LD}} \pm \sigma_{\text {stat }} \pm \sigma_{\text {syst }}[\mathrm{mas}]$ & $R \pm \sigma_{\text {tot }}\left[R_{\odot}\right]$ & $\chi_{\text {red }}^{2}$ \\
\hline$\tau$ Cet & B3-M0 & $K(2.179 \mu \mathrm{m})$ & 1.025 & $2.078 \pm 0.014 \pm 0.028$ & $0.816 \pm 0.013$ & 0.57 \\
\hline$\epsilon$ Eri & UT1-UT3 + B3-M0 & $K(2.179 \mu \mathrm{m})$ & 1.027 & $2.148 \pm 0.010 \pm 0.027$ & $0.743 \pm 0.010$ & 0.17 \\
\hline$\beta$ Leo & B3-M0 & $K(2.177 \mu \mathrm{m})$ & 1.014 & $1.449 \pm 0.014 \pm 0.023$ & $1.728 \pm 0.037$ & 1.32 \\
\hline$\beta$ Pic & UT1-UT3 & $K(2.177 \mu \mathrm{m})$ & 1.014 & $0.849 \pm 0.060 \pm 0.100$ & $1.759 \pm 0.241$ & 1.19 \\
\hline$\alpha$ PsA & UT1-UT3 + B3-M0 & $K(2.177 \mu \mathrm{m})$ & 1.014 & $2.222 \pm 0.021 \pm 0.015$ & $1.835 \pm 0.022$ & 0.41 \\
& E0-G1 & $K(2.177 \mu \mathrm{m})$ & 1.014 & $2.321 \pm 0.042 \pm 0.046$ & $1.917 \pm 0.054$ & 0.44 \\
& E0-G1 & $H(1.632 \mu \mathrm{m})$ & 1.016 & $2.235 \pm 0.056 \pm 0.046$ & $1.846 \pm 0.061$ & 0.15 \\
& All baselines & $K(2.177 \mu \mathrm{m})$ & 1.014 & $2.228 \pm 0.011 \pm 0.020$ & $1.840 \pm 0.023$ & 0.62 \\
\hline
\end{tabular}

a The linear limb-darkening coefficients were taken from Claret tables (Claret 2000) to compute the conversion factor $\rho$.

smaller number of scans: $\theta_{\mathrm{UD}}=2.289 \pm 0.042 \pm 0.046 \mathrm{mas}$ (i.e. 0.062 mas for a relative total error of $2.7 \%$ ).

These data can be dealt with in two different ways: we can either assume a circularly symmetric brightness distribution (Fig. 9) and make a general fit to the data (thus averaging the UV coverage on the azimuth angle), or fit different UD models on each baseline direction (Fig. 10), assuming an asymmetric brightness distribution. The first hypothesis leads to an equivalent UD diameter of $\theta_{\mathrm{UD}}=2.197 \pm 0.011 \pm 0.020$ mas (total error 0.022 mas $=1 \%$ ). The short spatial frequency observations do not constrain much the fit because of their large error. The probability for these visibilities to be well represented by a 2.197 mas UD model is still high: $88 \%\left(\chi_{\text {red }}^{2}=0.57\right)$ and therefore we cannot discriminate between a symmetric and an asymmetric brightness distribution from these perpendicular measurements. Table 3 summarizes the angular LD diameters and reduced chi-square values obtained for the two baselines orientations.

Finally, we mention the $H$ band measurement even if the visibility precision is lower compared to the $K$ band. The visibilities obtained on the E0-G1 baseline are coherent with the rest of the observations, and lead to an equivalent diameter $\theta_{\text {UD }}=2.199 \pm 0.055 \pm 0.045$ mas, a value compatible with the diameter obtained in the $K$ band with other baselines, within its uncertainty (the linear limb darkened coefficients are very similar, see hereafter).

\subsection{Limb darkened angular diameters and linear radii}

The UD model is not a correct representation of the real stellar profiles since it assumes a constant angular intensity of the surface brightness. It is therefore necessary to convert it into a limb darkened (LD) value, which is more representative of the actual brightness distribution.

Hanbury-Brown et al. (1974) have established an approximate formula linking the linear LD coefficient $u(\lambda)$ with the conversion factor $\rho$ defined by:

$\rho(\lambda)=\frac{\theta_{\mathrm{LD}}(\lambda)}{\theta_{\mathrm{UD}}(\lambda)}=\sqrt{\frac{1-u(\lambda) / 3}{1-7 u(\lambda) / 15}}$.

This approximation is satisfactory for our measurement since its associated error is not larger than $0.2 \%$, to be compared with our own minimum uncertainty of $1 \%$ on the fit UD values. We will follow the tables computed by Claret (2000) based on Kurucz models (Kurucz 1992), which provide the relevant coefficients for our spectral bands as a function of the turbulent velocity, effective temperature, surface gravity and metallicity.

The LD diameters can be finally converted into linear radii (in solar unit $R_{\odot}$ ) using the distance of the stars (cf. Table 1). Parallaxes are taken from the Hipparcos catalog (Perryman et al. 1997). The LD angular diameters and the corresponding radii are summarized in Table 3 .

\subsection{Discussion and comparison}

Stellar diameters are usually constrained by the estimated luminosity and effective temperature through the Stefan-Boltzmann relation. In order to improve this rough estimate, the relative error of direct measurements needs to be smaller than $5 \%$. The angular diameters inferred from our interferometric observations reach a 1 to $2 \%$ accuracy (including the systematics), except for $\beta$ Pic. The distance of these nearby dwarf stars being well known from the Hipparcos parallaxes, the final uncertainty on the linear radii is only slightly increased. 


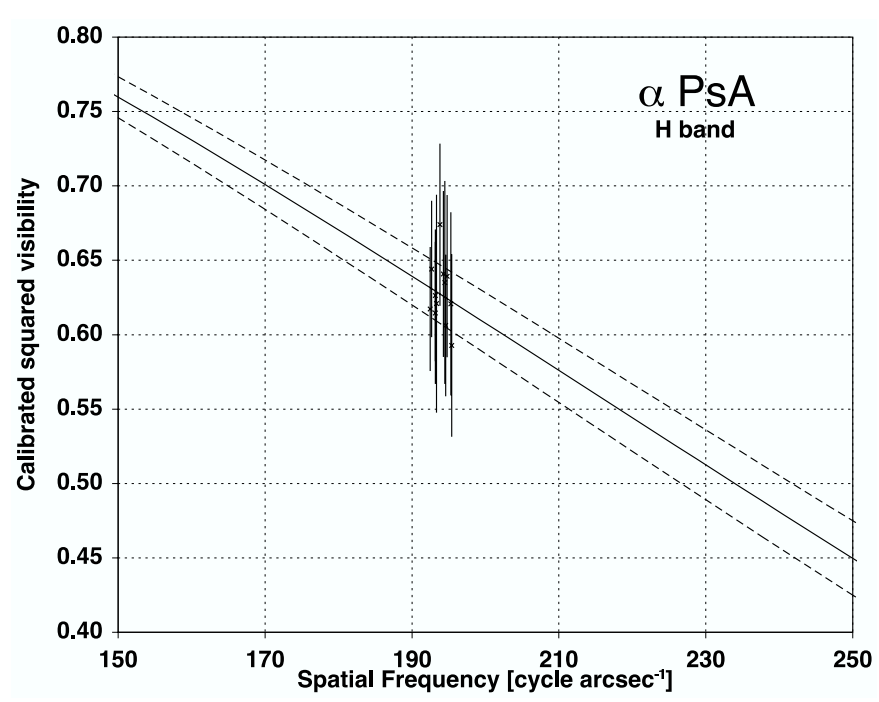

Fig. 11. $H$ band visibility measurements of $\alpha$ PsA with the E0-G1 baseline in July 2002 during the IONIC experiment. Despite the larger uncertainty on the transfer function estimate and on the visibility points, the data are consistent and the best UD model fit gives: $\theta_{\mathrm{UD}}=2.199 \pm 0.055 \pm 0.045$ mas, in good agreement with the $K$ band measurement.

The gain obtained with our direct measurements is visible in the Hertzsprung-Russell (HR) diagrams presented in Figs. 12 to 16 .

$\tau$ Ceti: The size of $\tau$ Cet has recently been estimated by Pijpers et al. (2003) from the same initial set of observations. They derived a radius $R=0.773 \pm 0.02 R_{\odot}$, which is smaller than our estimate. The discrepancy arises from the calibration of the raw visibilities. The authors used a single reference star ( $\theta$ Ceti), which has a low visibility on the $140 \mathrm{~m}$ long baseline. The estimation of the transfer function is consequently very sensitive to a slight change of its diameter. We chose, instead, to take into account a larger number of reference stars to minimize the systematic errors (as explained in Sect. 2.3) and checked the consistency of this main calibrator with other stars from Cohen's catalog. The revision of its angular diameter $(2 \sigma$ away from the value predicted by Cohen et al. 1999) turns into a $\sim 5 \%$ decrease for $V^{2}(\tau$ Cet $) \sim 35 \%$ on the $140 \mathrm{~m}$ baseline. We have also adopted a slightly shorter effective wavelength, based on the modeling of the overall transmission of the instrument and atmosphere at Paranal. It is important to notice how sensitive the $V^{2}$ normalization is to the quality of the calibrators, since the same set of data can lead to different astrophysical results. Indeed, we cannot confirm from our analysis the discrepancy observed by Pijpers et al. (2003) between the interferometric and spectroscopic diameters. This will be discussed further in Sect. 5.3. Both measurements are compared to the spectroscopic radius on the HR diagram plotted in Fig. 12.

$\epsilon$ Eridani: We have presented the first interferometric measurement obtained for this star. The VLTI radius is in excellent agreement with the spectrophotometric constraint, both values are compared on the HR diagram in Fig. 14.

$\beta$ Leonis: The angular diameter of this A3 dwarf has been first established by Hanbury-Brown et al. (1974) at the Narrabri
Interferometer but with a relative error of about $8 \%$. Their estimation in the visible led to $\theta_{\mathrm{LD}}=1.33 \pm 0.10$ mas, which is in agreement with (although slightly smaller than) the VLTI determination within its error bar. The total uncertainty has been reduced by a factor 4 . The discrepancy could be related to a possible non circularly symmetric brightness profile of the source (since the Narrabri observations were obtained at a different spatial frequency and probably different azimuth angles as well) or to a possible faint environment emitting in the infrared only. $\beta$ Leo has an intermediate rotational velocity, which may impact the shape of its photosphere and thus produce azimuth dependent visibilities. The consistency of the VLTI measurement with the spectroscopic radius is illustrated in Fig. 13.

$\beta$ Pictoris: Following the revised value of $\beta$ Pic's parallax by Hipparcos leading to a luminosity $\log \left(L / L_{\odot}\right)=0.944 \pm$ 0.012 (Crifo et al. 1997) and adopting an effective temperature $T_{\text {eff }}=8200 \pm 150 \mathrm{~K}$ (Lanz et al. 1995), one can derive a radius of $1.46 \pm 0.14 R_{\odot}$, using the Stefan-Boltzmann relation. Despite the low precision, it seems that we can at least conclude that the radius inferred from interferometry is slightly too large compared to what we would have expected. Our value is only consistent with the upper limit given by the spectroscopy (see Fig. 15). If the observed discrepancy is real and not biased by the reference stars used, we might either incriminate an effect of the photosphere (e.g., an equatorial oblateness induced by its rapid rotation) or the signature of the close stellar environment (dust, companion). In both cases, a better UV coverage is required to evidence unambiguously such effects and these possibilities are further discussed in the next section. Given the mean temperature of $8200 \mathrm{~K}$, an extinction of 0.15 to 0.6 mag is required to match the luminosities corresponding to our VINCI $1 \sigma$ error box. This is larger than the maximum extinction predicted by theoretical modeling of the dusty disk or estimation derived from multi-wavelengths observations (see discussion in Crifo et al. 1997).

$\alpha$ Piscinis Austrini: Like $\beta$ Leo, Fomalhaut was measured first by Hanbury-Brown et al. (1974), who derived a LD angular diameter $\theta_{\mathrm{LD}}=2.10 \pm 0.14$ mas from observations in the visible at $\lambda=4385$ and $4608 \AA$. Our own result is consistent with the Narrabri measurement, but far more precise. As mentioned in the previous section, our data set and its related precision do not unambiguously support an asymmetrical brightness distribution. Nevertheless, from the visibilities gathered on the $66 \mathrm{~m}$ baseline we cannot exclude a weak visibility modulation in this direction, parallel to the disk major axis and thus also presumably aligned with the stellar equatorial diameter. The effect of an hypothetical photospheric oblateness is discussed hereafter as well as the influence of a faint dusty ring. Figure 16 presents the VLTI constraint on the star's position in the HR diagram.

Figures 12 to 16 display in the HR diagram the comparison between our interferometric (thick tilted box) constraints on the stellar radii and the spectrophotometric domains (thin box) corresponding to the adopted photospheric parameters (cf. Table 4). Iso-radius lines are tilted and plotted for a few temperatures close to those expected, to provide $1 \sigma$ (solid) and $2 \sigma$ (dashed) VINCI error boxes. Filled diamonds correspond to the final evolution state from the modeling with 


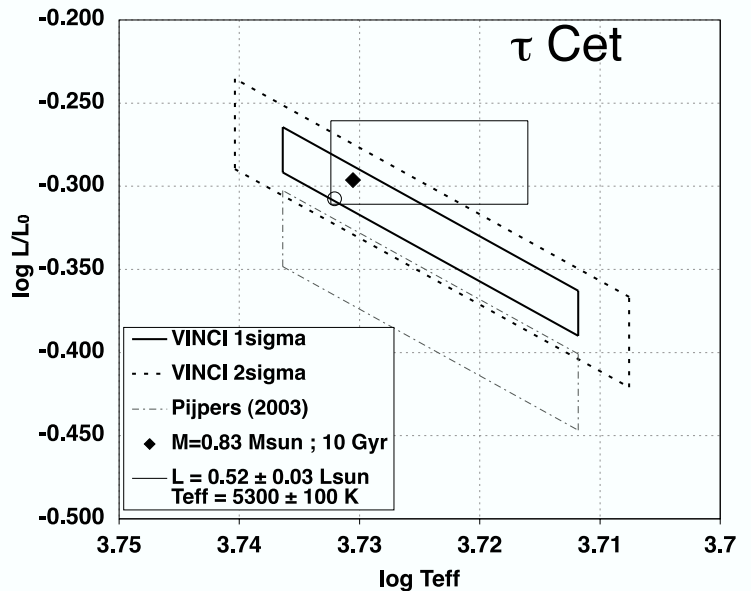

Fig. 12. Position of $\tau$ Ceti in the HR diagram (rectangle $=$ spectrophotometry, parallelograms are the VINCI error domains). The filled diamond corresponds to a model with $M=0.83 M_{\odot}, Z_{\mathrm{i}}=0.0145$, the open circle to $M=0.81 M_{\odot}$.

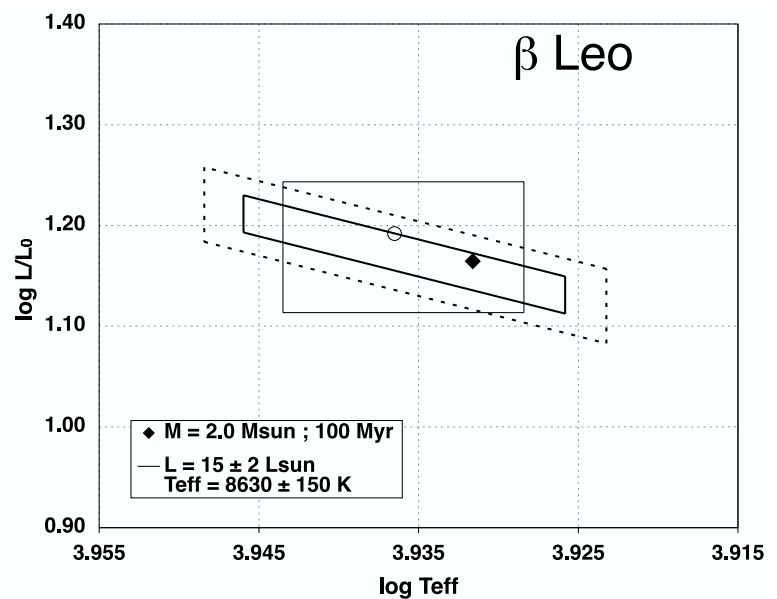

Fig. 13. Position of $\beta$ Leonis in the HR diagram. The filled diamond corresponds to $M=2.0 M_{\odot}, Z_{\mathrm{i}}=0.042$, the open circle to $M=$ $2.03 M_{\odot}$.

CESAM (Morel 1997), see Table 5. Increasing slightly the initial mass translates the star vertically and to the left, whereas increasing only the initial metallicity $\left(Z_{i}\right)$ makes it slide along the iso-radius line toward higher temperature. Open circles represent other tentative modeling described in Sect. 5. The present simulations show that we can reproduce both interferometric radius and spectroscopic characteristics with a maximum uncertainty of $2 \%$ on the mass (for a given initial helium and metal abundance).

\section{Constraining the circumstellar environment}

Mid-IR observations of the young prototype $\beta$ Pic (Lagage \& Pantin 1994; Pantin et al. 1997) have shown a clear depletion of dust in the inner disk (down to $10 \mathrm{AU}$ ), but no strict void of matter in the close vicinity of the star. More recently, the first observations of the Spitzer Space Telescope have also demonstrated the presence of warm dust around Fomalhaut

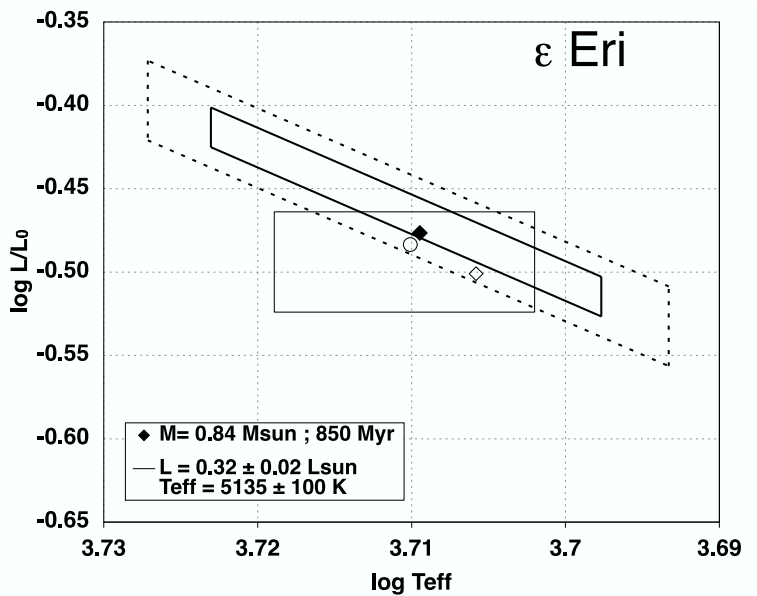

Fig. 14. Position of $\epsilon$ Eridani in the HR diagram. The filled diamond corresponds to $M=0.84 M_{\odot}, Z_{\mathrm{i}}=0.0215$, the open diamond to $M=$ $0.83 M_{\odot}, Z_{\mathrm{i}}=0.0215$ and the open circle to $M=0.83 M_{\odot}, Z_{\mathrm{i}}=0.02$.

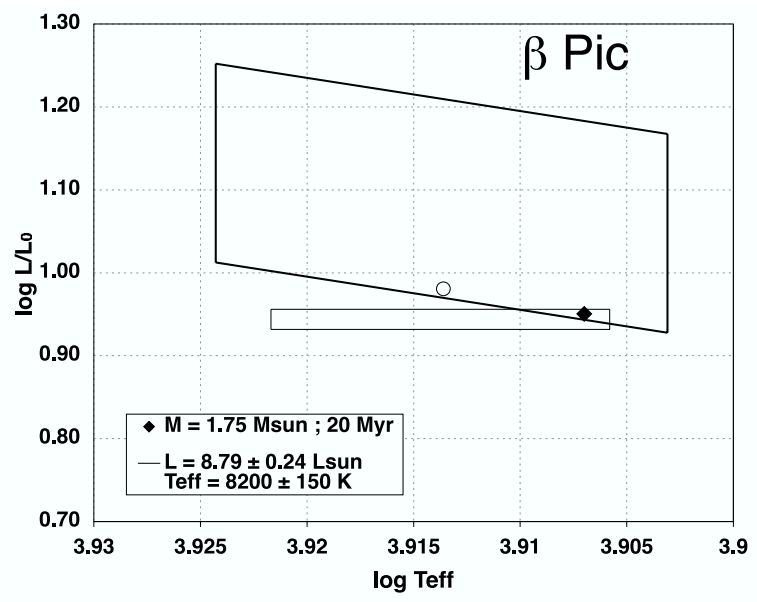

Fig. 15. Position of $\beta$ Pictoris in the HR diagram. The filled diamond corresponds to $M=1.75 M_{\odot}, Z_{\mathrm{i}}=0.0290$, the open circle to $M=1.77 M_{\odot}$. Due to the large uncertainty, only the $1 \sigma$ error box is displayed.

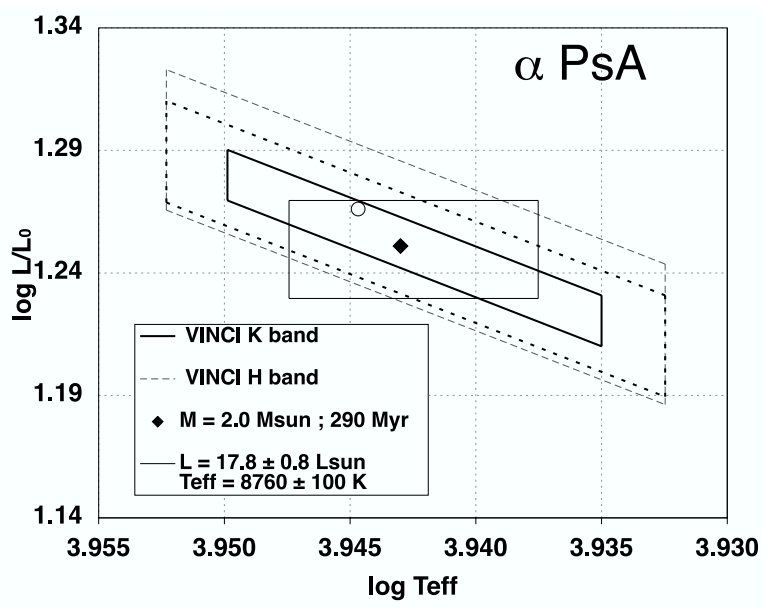

Fig. 16. Position of $\alpha$ PsA in the HR diagram. The filled diamond corresponds to $M=2.00 M_{\odot}, Z_{\mathrm{i}}=0.031$, the open circle to $M=2.02 M_{\odot}$. 
(Stapelfeldt et al. 2004). Images obtained at 24 and $70 \mu \mathrm{m}$ revealed the presence of warm grains in the first $100 \mathrm{AU}$, inside the central cavity observed at sub-mm wavelengths.

Near Fomalhaut, the dust produced by the collision cascade and comets evaporation is permanently removed by the radiation pressure and sublimation process. Although theoretical models do not expect large amounts of dust to be found in the inner system, observational constraints in the first AUs can only be inferred from photometric measurements and spectral energy distribution (SED) fitting, since imaging techniques do not provide yet the required spatial resolution. This method is limited by the photometric precision itself, as well as the ability for the stellar atmosphere codes (e.g., Kurucz models) to predict the photospheric flux in the considered spectral window.

With a typical accuracy of $1 \%$ at best, no significant nearIR excess has ever been reported so far around the selected targets and a 1 or $2 \%$ flux excess cannot be absolutely excluded. Actually, near-IR excesses are observed around old-PMS stars much younger than our targets but too faint compared to our current sensitivity (like HD 100546, see spectra obtained by Malfait et al. 1998). These stars are thought to show a transition phase in the disks evolution between young gaseous protoplanetary disks and second-generation dusty disks. From our interferometric observations, we can tentatively constrain the excess due to a putative warm environment, which is directly linked to the shape and precision of our visibility curves.

\subsection{Expected visibility from a simple modeling}

$K$ band interferometric instruments like VINCI or AMBER (Petrov 2000) on the VLTI should be able to detect even a small amount of dust in the first AUs around the star down to the sublimation distance. This distance can be estimated for refractory material from the grains absorption properties (see Monnier et al. 2002). Considering a wide range of grain sizes (from $\sim 0.1$ to a few microns), the corresponding sublimation distance around A stars ranges typically between 0.1 and $0.5 \mathrm{AU}$ (5 to 25 mas at the distance of $\beta$ Pic). At such radii, the dust luminosity, enhanced by the thermal emission of the particles, can reach a small fraction of the stellar luminosity at $2 \mu \mathrm{m}$. The detection threshold is determined by the precision of the interferometric measurement, since emission from an extended structure will produce a visibility modulation dependent on the grains size distribution and the geometry of the disk (or ring). The amplitude of the $V^{2}$ modulation is proportional to the contribution of the dust to the total brightness.

Figure 17 shows the expected visibility profile for a basic disk modeling, which consists of a thin ring observed face-on around a resolved star with $\theta_{\mathrm{UD}}=2$ mas. The exact shape of the $V^{2}$ modulation depends obviously on the radial extension of the ring, its inclination and the orientation of its major axis with respect to the baseline. For simplicity, it is located at $40 R_{\star}$, close to the sublimation distance and contributes to $2 \%$ of the total flux in the $K$ band. Detecting such a faint disk is challenging since it requires to evidence the faint departure from the Airy function of the stellar photosphere. The largest modulation amplitude can be observed at very low spatial frequency, indicating that the disk detection should be less difficult with very short baselines. In addition, a small visibility deficit (less than $1 \%$ ) can be observed even at high spatial frequencies, where VINCI data were collected (the frequency range was arbitrarily limited to the domain scanned by the VLTI).

\subsection{Debiasing from the rotation induced asymmetry}

Three of our program stars, namely $\beta$ Leo, $\beta$ Pic and $\alpha$ PsA, are reported in the literature to be rapid rotators. Their rotation speed collected from the Royer et al. (2002) catalog indicates an intermediate velocity: $v \sin i=128,130$ and $93 \mathrm{~km} \mathrm{~s}^{-1}$ respectively. This high rotation rate may elongate their atmosphere in the equatorial plane. The interferometric measurements along this direction could lead to larger equivalent UD diameters, compared to those measured in the perpendicular (polar) direction. Oblate photospheres have already been successfully evidenced by interferometric measurements (Van Belle et al. 2001; Domiciano et al. 2003) on much faster rotating stars (Altaï, $v \sin i \sim 190 \mathrm{~km} \mathrm{~s}^{-1}$ and Achernar, $\left.v \sin i \sim 225 \mathrm{~km} \mathrm{~s}^{-1}\right)$.

Using the Roche model of a rigid rotation body described by Domiciano et al. (2002), we have investigated the influence of the rotation on the visibility curves for two perpendicular baselines, in order to quantify the expected polar flattening for our targets. Results of the modeling are presented for $\beta$ Pic and $\alpha$ PsA in Fig. 18. The gravity darkening effect (Von Zeipel 1924), which makes the polar regions hotter than the equatorial zone, is taken into account for the most rapid rotator. We have plotted the $V^{2}$ difference between a polar and an equatorial measurement. For $\beta$ Leo, the orientation and inclination of its rotation axis are not known but the visibility modulation should be at least as large as that of $\beta$ Pic.

The modeling shows that, even for such intermediate rotation speeds, the oblateness can produce a visibility modulation as high as $\delta V^{2} \sim 3 \%$ in the case of $\beta$ Pic and $2 \%$ for Fomalhaut. With the projected baseline range covered by the VLTI data (see the arrows on the plots), the expected bias is in the order of $1 \%$. Even if this effect is not unambiguously detectable in the reported observations because of our lack of precision, it could produce a bias in our analysis. Assuming that the detected dusty disks around these stars lie into their equatorial plane, the UV coverage indicates that Fomalhaut was measured in the polar direction with the long baselines and close to the equator with the $66 \mathrm{~m} \mathrm{E0-G1} \mathrm{(see} \mathrm{Fig.} \mathrm{8).} \mathrm{As} \mathrm{for} \beta$ Pic, the UTs observations are almost aligned with the disk major axis. The modeled deficit should then be subtracted to the measured visibilities before estimating the constraint on the disk flux itself. As a consequence, the derived upper values for the flux will depend on the relevance of the modeling. Only observations at low spatial frequency are not affected by the polar flattening.

\subsection{Constraint on the disk brightness and consequence}

Once Fomalhaut and $\beta$ Pic visibility data are corrected from the expected bias, the maximum disk brightness can be estimated 


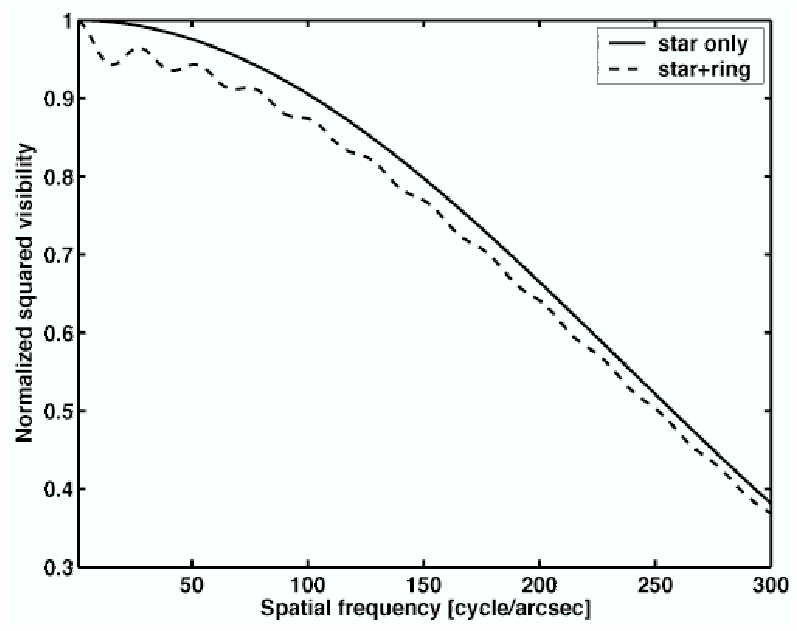

Fig. 17. Simulated visibility profiles for a 2 mas UD diameter star with no environment (solid line) or surrounded by a faint and narrow uniform ring $\left(F^{\text {ring }}=0.02 F^{\text {tot }}\right)$ located at 40 stellar radii (dashed line). The departure from the Airy function reaches about 5\% at very low spatial frequency whereas the curve can be approximated in the high frequency domain by the Airy function of a slightly bigger star.
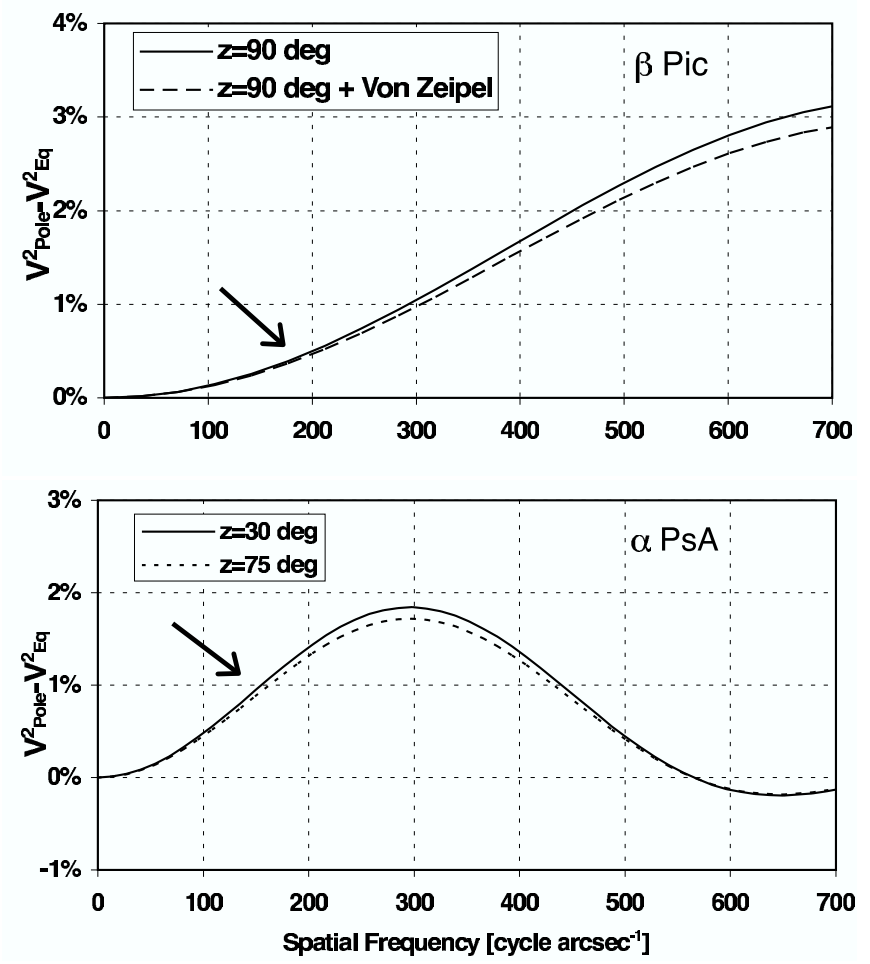

Fig. 18. Oblateness of $\beta$ Pic $\left(R_{\text {Pole }}=1.53 R_{\odot}, v \sin i=130 \mathrm{~km} \mathrm{~s}^{-1}\right)$ and $\alpha$ PsA $\left(R_{\text {Pole }}=1.83 R_{\odot}, v \sin i=93 \mathrm{~km} \mathrm{~s}^{-1}\right)$. Predicted $V^{2}$ difference between measurements along the polar and equatorial directions for various inclinations $(z)$ of the rotation axis. The influence of the gravity-darkening (VZ) is also shown in the case of $\beta$ Pic. The arrows indicate the spatial frequency of our interferometric measurements.

from the largest visibility deficit allowed by our measurements. As shown in Fig. 17, the constraint will arise from the short baseline observations since the visibilities at the covered high frequencies can hardly be distinguished from an Airy function. Actually, the quantity that we must consider is the difference

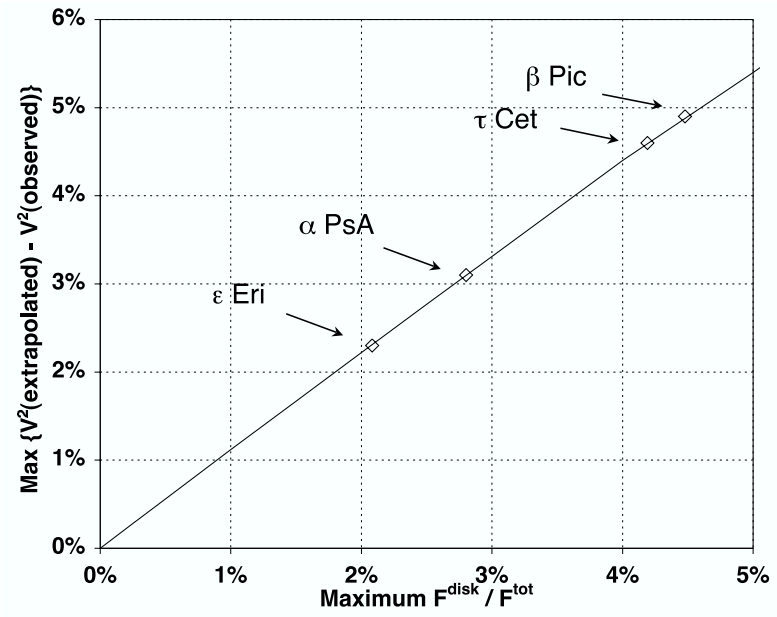

Fig. 19. Maximum dust/stellar flux ratio derived from the visibility measurements at different spatial frequencies, after correction of the expected oblateness for the fast rotating stars. The constraint on the presence of hot dust in a ring is only slightly weaker than that inferred from spectroscopic measurements.

between the measured visibility at $66 \mathrm{~m}$ and the extrapolation of the best UD model fit to the $140 \mathrm{~m}$ data (in the case of $\beta$ Pic, we can alternatively use the minimum diameter derived from photometry). Figure 19 shows this evolution as a function of the disk brightness for the simple model of dust ring mentioned previously. The measured maximum $V^{2}$ deficit consistent with our observations is reported on the plot for $\tau$ Ceti, $\epsilon$ Eri, $\beta$ Pic and $\alpha$ PsA

Even if the current precision of our instrument does not allow us to detect unambiguously the disk or to put severe constraints on its brightness, the modeling indicates that its presence might induce a possible bias on the stellar diameter determination. Indeed, from measurements in a limited range of spatial frequencies with a typical $1-2 \%$ accuracy, the inferred diameter might be too large due to the weak visibility decrease induced by the ring. Computations show that the relative bias on the equivalent UD diameter is of the order of the excess due to the putative disk, which could be up to $1-2 \%$ and thus similar to the total uncertainty on our diameter measurements. For comparison, previous interferometric observations of Vega with PTI (Ciardi et al. 2001) at high spatial frequency were found to be consistent with the presence of a warm debris disk contibuting for 3 to $6 \%$ of the stellar flux. The corresponding stellar diameter was reduced by $2-2.5 \%$ compared to that derived from a simple photospheric model without disk.

\section{Modeling the physical parameters of the stars}

Taking advantage of our new constraints on these stellar radii, we will further investigate the physical parameters of these stars and build coherent models in agreement with the spectroscopic quantities. With the help of evolution models, one can in particular estimate the age of a star for a given mass, luminosity, effective temperature and initial chemical abundance. In this section, we propose the results of modeling our five candidates with the stellar evolution code CESAM (Morel 1997). 
Table 4. Adopted stellar fundamental parameters.

\begin{tabular}{lccccccc}
\hline \hline Star & $\log \left(L / L_{\odot}\right)$ & $T_{\text {eff }}(\mathrm{K})$ & Age $(\mathrm{Myr})$ & $M / M_{\odot}$ & $R^{\mathrm{VINCl}} / R_{\odot}$ & $\log g\left(\mathrm{~cm} \mathrm{~s}^{-2}\right)$ & {$[\mathrm{Fe} / \mathrm{H}](\mathrm{dex})$} \\
\hline$\tau$ Cet & $-0.291 \pm 0.030$ & $5264 \pm 100$ & $4400-12000$ & $0.85 \pm 0.15$ & $0.816 \pm 0.013$ & $4.5 \pm 0.2$ & -0.5 \\
$\epsilon$ Eri & $-0.493 \pm 0.030$ & $5135 \pm 100$ & $700 \pm 200$ & $0.90 \pm 0.10$ & $0.743 \pm 0.010$ & $4.7 \pm 0.2$ & $-\mathbf{0 . 0 7}+0.06$ \\
$\beta$ Leo & $1.176 \pm 0.058$ & $8630 \pm 150$ & $100-380$ & $1.78 \pm 0.46$ & $1.728 \pm 0.037$ & $4.2 \pm 0.1$ & 0.00 \\
$\beta$ Pic & $0.944 \pm 0.012$ & $8200 \pm 150$ & $8-280$ & $1.75 \pm 0.10$ & $1.759 \pm 0.241$ & $4.2 \pm 0.1$ & -0.02 \\
$\alpha$ PsA & $1.250 \pm 0.020$ & $8760 \pm 100$ & $200 \pm 100$ & $2.00 \pm 0.20$ & $1.840 \pm 0.023$ & $4.2 \pm 0.1$ & -0.03 \\
\hline
\end{tabular}

Table 5. Stellar fundamental parameters from the modeling with CESAM. "s" means at surface.

\begin{tabular}{lcccccccc}
\hline \hline Star & $\log \left(L / L_{\odot}\right)$ & $T_{\text {eff }}(\mathrm{K})$ & Age $(\mathrm{Myr})$ & $M / M_{\odot}$ & $R / R_{\odot}$ & $\log g\left(\mathrm{~cm} \mathrm{~s}^{-2}\right)$ & {$[\mathrm{Fe} / \mathrm{H}]_{\mathrm{s}}$} & $\overline{\Delta v_{0}}(\mu \mathrm{Hz})$ \\
\hline$\tau$ Cet & -0.296 & 5377 & 10000 & 0.83 & 0.821 & 4.53 & -0.31 & 169 \\
$\epsilon$ Eri & -0.476 & 5122 & 850 & 0.84 & 0.735 & 4.63 & -0.06 & 205 \\
$\beta$ Leo & 1.165 & 8542 & 100 & 2.00 & 1.748 & 4.25 & 0.06 & 78 \\
$\beta$ Pic & 0.950 & 8073 & 20 & 1.75 & 1.53 & 4.31 & -0.04 & 89.5 \\
$\alpha$ PsA & 1.247 & 8751 & 290 & 2.00 & 1.832 & 4.21 & -0.10 & 72.8 \\
\hline
\end{tabular}

Our simulations are compared to the observed parameters in Table 5.

\subsection{Estimating the fundamental parameters using CESAM}

None of our targets is a known gravitationally bound binary system and hence no precise estimate of the stellar mass is available. We have built coherent models with estimated initial masses derived from the spectroscopy. The computation must converge in a luminosity-temperature box at a given age (see HR diagrams in Figs. 12 to 16).

The evolutionary models of the program stars were computed using the CESAM code version 4 (Morel 1997) including the pre-main sequence evolution. The ordinary assumptions of stellar modeling are made, i.e. spherical symmetry, no rotation, no magnetic field, no mass loss. The relevant nuclear reaction rates are taken from the NACRE compilation (Angulo et al. 1999). The equation of state adopted is EFF (Eggleton et al. 1973), and the OPAL opacities are from Iglesias \& Rogers (1996) with the Grevesse \& Noels (1993) mixture. Microscopic diffusion is described using the formalism of Burgers (1969) with resistance coefficients from Paquette et al. (1986). We take into account radiative diffusivity as recommended by Morel \& Thévenin (2002), that limits the efficiency of microscopic diffusion in outer layers of stars with intermediate masses like $\beta$ Pic and $\alpha$ PsA. We have neglected radiative accelerations, as well as the changes of abundance ratios between metals within $Z$. The atmosphere is restored using Hopf's $T(\tau)$ law (Mihalas 1978). The adopted radius of the star is the bolometric radius, where $T\left(\tau_{\star}\right)=T_{\text {eff }}$ in the model. In the convection zones the temperature gradient is computed according to the MLT $_{\mathrm{CM}}$ convection theory with a mixing length parameter of $\Lambda=1$ (Canuto \& Mazzitelli 1991, 1992). Following the prescriptions of Schaller et al. (1992) we have computed models that include convective core overshoot (radius $R_{\mathrm{co}}$ ) over a distance $O_{\mathrm{v}}=0.15 \min \left(H_{\mathrm{p}}, R_{\mathrm{co}}\right)$ for the three A type stars and $O_{\mathrm{v}}=0.0$ for the other two stars $\epsilon$ Eri and $\tau$ Ceti. These adopted overshoot values are in agreement with Ribas, Jordi \& Carme (2000) for a $2 M_{\odot}$ star. Our modeling does not take into account the influence of rotation on stellar internal structure, a condition that may impact the mixing and thus the surface abundance.

The initial helium content $Y_{i}$ of the program stars is unknown. This parameter strongly correlates with the value of stellar radius. Its choice however is not free and obeys to a galactic enrichment of helium starting from $Y_{i}=0.24$ at the age of the Big Bang. Adopting this primordial value of Izotov et al. (1997), Pagel \& Portinari (1998) have deduced a ratio $\Delta Y / \Delta Z=2-3$ of fresh helium supplied to the interstellar medium by stars. Then for a metal-poor star like $\tau$ Ceti we can adopt an initial $Y_{\mathrm{i}}=0.25-0.26$ and a presumably very young star like $\beta$ Pic has probably a higher value $Y_{\mathrm{i}}=0.27-0.29$. This enrichment law of the helium content is still debated and nothing is clear today, as regard to the Hyades, which do not fit this law with a $Y_{\mathrm{i}}=0.255$ and a young age of $600 \mathrm{Myr}$.

\subsection{Predicting the mean large frequency spacing}

From our modeling, it is possible to derive one of the main observables used in asteroseismic studies and related to the stellar density. Asteroseismology aims at probing the internal structure and the fundamental parameters of the stars through the study of their normal-mode pulsations, excited at the basis of the convective layer in their atmosphere. Because of their spherical geometry, acoustic oscillation eigenmodes can be conveniently written as the product of a function of radius and a spherical harmonic. The mean large frequency spacing (or large separation $\overline{\Delta v_{0}}$ ), inferred from the high frequency domain of the oscillation spectrum, is defined as the average of differences between frequencies of modes with consecutive radial orders $(n)$. It is linked to the mean stellar density, following the relation: $\overline{\Delta v_{0}} \sim 135 \sqrt{\left(M_{\star} / M_{\odot}\right) /\left(R_{\star} / R_{\odot}\right)^{3}} \mu \mathrm{Hz}$ (Brown \& Gilliland 1994). Combining asteroseismic and interferometric observations may thus allow us to determine precise 
estimations of the mass of the stars, one of the main parameters that govern their evolution. In the next sections, we predict from our modeling the expected large separation for our five targets. Instruments like HARPS at ESO or the space missions MONS and COROT will soon make it possible to determine these frequencies with high precision (down to $1 \mu \mathrm{Hz}$ ).

\section{3. $\tau$ Ceti}

The main difficulty in constructing a coherent model of the G8 dwarf star $\tau$ Ceti consists in the choice of its initial metallicity $Z$ and its mass $M$. Indeed, the determination of iron abundance (Santos et al. 2001) indicates that $\tau$ Ceti is a moderate metal-poor star of the old disk of the Galaxy and consequently the well-known $\alpha$ element enhancement must be taken into account. For an old disk star of -0.5 dex of metallicity, the mean trend ratio of alpha elements $[\alpha / \mathrm{Fe}]$ is about 0.2 dex. Therefore, an element like $\mathrm{O}$ is supposed to be enhanced by a factor 1.7, and thus increases the stellar interior opacity. Soubiran et al. (1998) derived a metal abundance of -0.5 dex with $T_{\text {eff }}=5300 \pm 100 \mathrm{~K}$. Thévenin (1998), adopting $T_{\text {eff }}=5250 \mathrm{~K}$, derived from a detailed abundance analysis $[\mathrm{Fe} / \mathrm{H}]=-0.42$. Increasing slightly the temperature increases also the derived abundance and therefore we prefer to adopt $[\mathrm{Fe} / \mathrm{H}]=-0.4$ dex for the iron abundance with an effective temperature of $5300 \mathrm{~K}$. Then, taking into account the enhancement of $\alpha$ elements abundance suggests us to adopt an initial metallicity of $\left(\frac{Z}{X}\right)_{i}=0.0145$ corresponding to $[\mathrm{Fe}+\alpha / \mathrm{H}]_{\mathrm{i}}=-0.23$ and $Y_{\mathrm{i}}=0.250$ (close to the primordial value of 0.24 , corresponding to an helium enrichment of the Galaxy of 0.01 since the Big Bang for a star of $10 \mathrm{Gyr}$ ).

After $10 \mathrm{Gyr}$ of time evolution, adopting an initial mass of $M=0.83 M_{\odot}$, the surface abundance is reduced to $\left(\frac{Z}{X}\right)_{i}=$ 0.0120 (after element diffusion). It corresponds to a surface metallicity $[\mathrm{Metal} / \mathrm{H}]_{\mathrm{s}}=\log (Z / X)-\log (Z / X)_{\odot}=-0.31$ with $(Z / X)_{\odot}=0.0245$. The simulation converges with a luminosity $\log \left(L / L_{\odot}\right)=-0.296$ (to be compared with $\log \left(L / L_{\odot}\right)=$ $-0.284 \pm_{0.026}^{0.024}$, Pijpers et al. 2003), an effective temperature $T_{\text {eff }}=5377 \mathrm{~K}$ and a linear radius of $0.821 R_{\odot}$ in agreement with the VLTI determination. The final age agrees with the estimation derived by Lachaume et al. (1999), who found it in the range 4.4-12Gyr, based on the analysis of the CaII emission lines. We note that a model adopting a metallicity around -0.5 , as derived by Soubiran et al. (1998), leads to a larger age $(\sim 10.4 \mathrm{Gyr})$ and higher temperature $(\sim 5550 \mathrm{~K})$, which seems less probable on the view of the various published $T_{\text {eff }}$ in the literature. Our modeling adopts an initial helium content $Y_{\mathrm{i}}=0.25$ close to the primordial value. Choosing, instead, $Y_{\mathrm{i}}=0.265$ requires shifting the mass to $0.81 M_{\odot}$, resulting at $9.5 \mathrm{Gyr}$ in $T_{\text {eff }}=5396 \mathrm{~K}, \log \left(L / L_{\odot}\right)=$ -0.308 and $R=0.802 R_{\odot}$ (see open circle in the HR diagram, Fig. 12). This solution, on the edge of the error box, is less probable and we finally adopt the first model with a primordial helium abundance. Other derived parameters are summarized in Table 5 .

Pijpers et al. (2003) have chosen to adopt a larger helium content but with a much smaller mass $\left(0.77 M_{\odot}\right)$ to account for both spectroscopic observations and their interferometric measurement. It results (for the model based on the interferometric radius) in a temperature larger than $5500 \mathrm{~K}$, which seems unlikely, as discussed previously. The authors predict for the large separation the value $173 \mu \mathrm{Hz}$, which is very close to our own estimation: $\overline{\Delta v_{0}} \sim 169 \mu \mathrm{Hz}$. One must however keep in mind that both models assume different values for the mass and radius (their variations actually almost compensate in the computation of the large separation). Observations with HARPS at La Silla should soon provide an independent estimation of this parameter and make it possible to build a more precise model of this star.

\section{4. $\epsilon$ Eridani}

The very nearby Sun-like star $\epsilon$ Eridani is a chromospherically active star. Its mass is estimated in the range $0.9 \pm 0.1 M_{\odot}$ and its effective temperature around $5100 \mathrm{~K}$. With $M_{\text {bol }}=5.982 \pm 0.03$, adopting a mass of $0.84 M_{\odot}$, we have obtained a model with consistent values for the luminosity and effective temperature (see Table 5). The simulation converges toward a radius $R=0.735 R_{\odot}$, in excellent agreement with our interferometric measurement (see the filled diamond in the HR diagram, Fig. 14). The corresponding age is $850 \mathrm{Myr}$ for a metallicity $[\mathrm{Fe} / \mathrm{H}]_{\mathrm{s}}=-0.06$ and the large spacing frequency is predicted to be $\overline{\Delta v_{0}} \sim 205 \mu \mathrm{Hz}$. Observational data on its metallicity show a large range of possible values and we have adopted the recent iron abundance determination by Bodaghee et al. (2003).

Because there is no consensus about its metallicity and mass we have built a second model, which led, at the same age, to a metal abundance of -0.09 dex with a smaller mass of $0.83 M_{\odot}$. We derived a smaller diameter $\left(0.727 R_{\odot}\right)$. This value is just below the limit of our interferometric measurement and the first model is preferred. This dwarf star is believed to be older than the two other prototypes $\alpha$ PsA and $\beta$ Pic, with an age similar to the Hyades (800 Myr for Henry et al. 1996, from CaII lines; $730 \pm 200 \mathrm{Myr}$ for Song et al. 2000, from Li abundance, position in the H-R diagram and kinematics). Even if our model does not represent a unique solution (due to the unknown mass and exact metallicity), it is successful in accounting for the adopted parameters and confirms that $\epsilon$ Eri is substantially older than $\alpha$ PsA and $\beta$ Pic.

\section{5. $\beta$ Leonis}

$\beta$ Leo is a relatively young $\mathrm{A} 3 \mathrm{~V}$ star with a faint debris disk. Its spectroscopic parameters have been chosen following Decin et al. (2003) and we have built two models in good agreement with our constraints. Adopting an initial helium content $Y_{\mathrm{i}}=$ $0.295, Z_{\mathrm{i}}=0.042$ and a mass of $2.00 M_{\odot}$, the evolution converges in $100 \mathrm{Myr}$ toward $T_{\text {eff }}=8542 \mathrm{~K}, \log \left(L / L_{\odot}\right)=1.165$ and $R=1.748 R_{\odot}$ (filled diamond in Fig. 13).

Decreasing its age would shift the star horizontally in the HR diagram toward higher temperatures and it would result in a smaller radius but an increased metallicity $\left([\mathrm{Fe} / \mathrm{H}]_{\mathrm{s}}=0.06\right.$ in the present case). The sensitivity to the initial stellar mass is illustrated by the open circle corresponding to $M=2.03 M_{\odot}$, 
with the same initial abundances and age. Our final age agrees with the lower limit derived by Lachaume et al. (1999), who found it in the range 100-380 Myr based on isochrones fitting. From our simulations, it appears difficult to obtain an age larger than $200 \mathrm{Myr}$ with our constraints.

\section{6. $\beta$ Pictoris}

We adopted for this A5 dwarf star an initial mass of $1.75 M_{\odot}$, following Crifo et al. (1997) $\left(1.75 \pm 0.05 M_{\odot}\right)$. Lanz et al. (1995) and Holweger et al. (1997) argued that they fully reproduce the UV spectrum of the star and spectral lines of absorption with a solar abundance and an effective temperature of $8200 \mathrm{~K}$. The detailed abundance analysis by Holweger et al. (1997) revealed a mean metallicity close to the solar one within a factor two. Individual abundances of elements do not seem to depart strongly from the solar mixture. Therefore we adopt $\left(\frac{Z}{X}\right)_{\mathrm{i}}=0.0290$, which corresponds to an initial surface iron abundance $[\mathrm{Fe} / \mathrm{H}]_{\mathrm{i}}=+0.08$, expecting that at the age of the star, the surface abundance is close to the solar one because of the gravitational settlement. We do not take into account individual element abundance variations from the adopted metallicity because of the rather large uncertainties on the estimated abundances. The initial helium content is fixed to $Y_{\mathrm{i}}=0.280$, according to the enrichment law mentioned previously.

Despite its large uncertainty, our interferometric measurement forces the model to generate a quite large diameter. The computed luminosity and effective temperature are $\log \left(L / L_{\odot}\right)=0.950$ and $T_{\text {eff }}=8073 \mathrm{~K}$, both within the error bars of the adopted observed values, and corresponding to a radius of $1.53 R_{\odot}$ consistent with our measurement. The surface abundance is $[\mathrm{Fe} / \mathrm{H}]_{\mathrm{s}}=-0.04$ after $20 \mathrm{Myr}$ of evolution. There has been a debate on the age of $\beta$ Pic, the estimations varying from 8 to $300 \mathrm{Myr}$. Recent works favor the lowest values. Barrado y Navascués et al. (1997) inferred an age of $20 \pm 10 \mathrm{Myr}$, studying two comoving low mass stars. Zuckerman et al. (2001) estimated that it may be between 8 and $20 \mathrm{Myr}$ old (from a larger sample of 17 coeval stars in the Beta Pictoris Moving Group (BPMG) on the basis of their positions in the H-R diagram and on the time compatible with the Lithium depletion). This extreme youth is also supported by Ortega et al. (2002), whose estimation is based on the analysis of stellar velocities among the BPMG. They derived an age of $11.5 \mathrm{Myr}$, in good agreement with previous lower limit estimations. Our estimation confirms this suspected youth, which can also be correlated with the presence of the most massive disk among Vega-type stars, even including a stable gaseous component.

We noticed nevertheless that the radius at the age of $20 \mathrm{Myr}$ derived from our simulation agrees only with the lower limit of the VLTI measurement. From our analysis it appears difficult to build a model of $\beta$ Pic's atmosphere leading to a much larger diameter and reproducing correctly the spectroscopic quantities: we would have indeed to increase its mass and thus to consider a higher luminosity. Another tentative modeling with $M=1.85 M_{\odot}$ led to a diameter of $1.63 R_{\odot}$ after $45 \mathrm{Myr}$ of evolution (open circle in Fig. 15). A luminosity as large as $10 L_{\odot}$ is however required to support this computation, which is unlikely or requires an improbable enhanced extinction (at least $0.15 \mathrm{mag}$ ). Therefore, the first scenario is preferred and we adopt from this theoretical work a radius close to $1.53 R_{\odot}$. We should emphasize that this simulation assumes that the VLTI measurement is not affected by the presence of warm dust or by a companion, two conditions that could bias (toward higher values) the apparent size of the photosphere.

\section{7. $\alpha$ Piscinis Austrini}

The mass of the A3 dwarf $\alpha$ PsA is also poorly constrained: estimations in the literature vary around $2.1 \pm 0.2 M_{\odot}$. The simulation was initialized with $M=2.00 M_{\odot}$ and an initial abundance $\left(\frac{Z}{X}\right)_{i}=0.031$, corresponding to $[\mathrm{Fe} / \mathrm{H}]_{\mathrm{i}}=0.10$. The helium content is arbitrarily chosen as $Y_{\mathrm{i}}=0.290$. The star is supposed to have a surface abundance after evolution and diffusion of elements in agreement (within the error bars) with the value found for its associated companion Gl $879(-0.11 \pm 0.2 \mathrm{dex})$. The simulation converges toward a radius $R=1.832 R_{\odot}$ at $290 \mathrm{Myr}$ and a surface abundance $[\mathrm{Fe} / \mathrm{H}]=-0.10$. The derived diameter corresponds to a temperature $T_{\mathrm{eff}}=8751 \mathrm{~K}$ with a luminosity $L=17.66 L_{\odot}$ and is compatible with our interferometric diameter measurement on the long UTs and siderostats baselines. The age of Fomalhaut has been estimated by Barrado y Navascués (1997) thanks to its presumably comoving companion Gl 879. An age of $200 \pm 100 \mathrm{Myr}$ has been established from various methods such as the analysis of the Lithium abundance, the rotation speed, the stellar activity and it turned out that the star should be significantly older than the Pleiades. A similar estimation was obtained by Lachaume et al. (1999) with an age bracketed between 105 and $340 \mathrm{Myr}$, in good agreement with our simulation.

\section{Conclusion and perspective}

We have reported in this paper the first VLTI observations of debris disk stars. The stars $\beta$ Pictoris and $\epsilon$ Eridani have been resolved for the first time, and we have improved by a significant factor our knowledge of the fundamental parameters for the three other targets. Modeling will further benefit from the coupled interferometric and asteroseismic observations to determine precise estimations of the age and mass of the stars. Three of our measured stellar radii ( $\tau$ Cet, $\epsilon$ Eri and $\alpha$ PsA) have been used together with other VINCI measurements of MS dwarf stars to calibrate their surface brightness relations (Kervella et al. 2004b). This work is especially useful to extend and refine the reference star catalogs for long baseline interferometry.

Our analysis of the VINCI visibilities shows that the current instrumental precision does not allow us yet to detect unambiguously the signature of a small amount of warm dust close to the selected stars. Further measurements at lower spatial frequencies should however make it soon possible. Nevertheless, our analysis of the present data provides upper limits on the dust contribution to the total flux, which are of the same order of magnitude as those derived from the photometry and should be much improved in the near future thanks to the incoming 
fringe trackers. We have also pointed out that the possible confusion with photospheric effects strengthens the need for accurate and validated models of fast rotating stellar atmospheres. Visibility measurements at various IR wavelengths may help disentangle the respective contribution of such effects that can bias the detection of CS material. Finally we showed that even a faint disk that cannot be detected could potentially bias our diameter estimations by at most $1-2 \%$, depending on its relative flux contribution.

Future observations with the VLTI will benefit from increased sensitivity with the use of Auxiliary Telescopes and adaptive optics on the Unit Telescopes, thus giving access to a large sample of Vega-like stars and also to younger stars surrounded by transition disks easier to detect. The first generation of scientific instruments, namely MIDI (Leinert et al. 2000) in the thermal IR and AMBER (Petrov et al. 2000) in the nearIR will tackle the planet formation scenarii. Thanks to the implementation of fringe tracking facilities (like FINITO and PRIMA/FSU), the instrumental precision in the $K$ band is expected to be improved significantly and thus will make possible the detection of faint dusty disks or rings in the first AU around these stars.

Acknowledgements. These interferometric measurements have been obtained using the VLTI, operated by the European Southern Observatory at Cerro Paranal, Chile. We thank the VLTI Team (Garching and Paranal) for their essential support in this research. All the VINCI data presented in this letter are public and were retrieved from the ESO/ST-ECF Archive (Garching, Germany). The authors wish to acknowledge the anonymous referee for his/her precious help in improving the quality and readability of this paper. D.S. acknowledges the support of the Swiss FNRS. This research has made use of the SIMBAD database at CDS, Strasbourg (France).

\section{References}

Angulo, C., Arnould, M., Rayet, M., et al. 1999, Nucl. Phys. A, 656, 3 Artymowicz, P., \& Clampin, M. 1997, ApJ, 490, 863

Aumann, H. H., Beichman, C. A., Gillett, F. C., et al. 1984, ApJ, 278, L23

Aumann, H. H. 1985, PASP, 97, 885

Backman, D. E., Gillett, F. C., \& Low, F. J. 1986, AdSpR, 6, 43

Barrado y Navascués, D., Stauffer, J. R., Hartmann, L., \& Balachandran, S. C. 1997, ApJ, 475, 313

Barrado y Navascués, D. 1998, Ap\&SS, 263, 239

Bodaghee, A., Santos, N.C., Israelian, G., \& Mayor, M. 2003, A\&A, 404,715

Bordé, P., Coudé du Foresto, V., Chagnon, G., \& Perrin, G. 2002, A\&A, 393, 183

Brown, T. M., \& Gilliland, R. L. 1994, ARA\&A, 32, 37

Burgers, J. M. 1969, Flow Equations for Composite Gases (New York Academic Press)

Canuto, V. M., \& Mazitelli, I. 1991, ApJ, 370, 295

Canuto, V. M., \& Mazitelli, I. 1992, ApJ, 389, 729

Cayrel de Strobel, G., Hauck, B., Frano̧is, et al. 1992, A\&AS, 95, 273

Ciardi, D. R., van Belle, G. T., Akeson, R. L., et al. 2001, ApJ, 559, 1147

Claret, A. 2000, A\&A, 363, 1081

Cohen, M., Walker, R. G., Carter, B., et al. 1999, AJ, 117, 1864

Crifo, F., Vidal-Madjar, A., Lallement, R., Ferlet, R., \& Gerbaldi, M. 1997, A\&A, 320, L29
Decin, G., Vandenbussche, B., Wælkens, C., et al. 2003, ApJ, 598, 636

Decin, G., Dominik, C., Waters, L. B. F. M., et al. 2003, ApJ, 598, 636

Dent, W. R. F., Walker, H. J., Holland, W. S., \& Greaves, J. S. 2000, MNRAS, 314, 702

Domiciano de Souza, A., Vakili, F., Jankov, S., et al. 2002, A\&A, 393, 345

Domiciano de Souza, A., Kervella, P., Jankov, S., et al. 2003, A\&A, 407, L47

Eggleton, P. P., Faulkner, J., \& Flannery, B. P. 1973, A\&A, 23, 325

Glindemann, A., Abuter, R., Carbognani, F., et al. 2000, SPIE, 4006, 2

Greaves, J. S., Holland, W. S., Moriarty-Schieven, G., et al. 1998, ApJ, 506, L133

Greaves, J. S., Wyatt, M. C., Holland, W. S., \& Dent, W. R. F. 2004, MNRAS, 351, L54

Grevesse, N., \& Noels, A. 1993, Cosmic Abundances of the Elements, in Origin and Evolution of the Elements, ed. N. Prantzos, E. Vangioni-Flam, \& M. Cassé (Cambridge University Press), 14

Hanbury Brown, R., Davis, J., \& Allen, L. R. 1974, MNRAS, 167, 121

Henry, T. J., Soderblom, D. R., Donahue, R. A., \& Baliunas, S. L. 1996, AJ, 111, 439

Holland, W. S., Greaves, J. S., Zuckerman, B., et al. 1998, Nature, 392, 788

Holland, W. S., Greaves, J. S., Dent, W. R. F., et al. 2003, ApJ, 582, 1141

Holweger, H., Hempel, M., van Thiel, T., \& Kaufer, A. 1997, A\&A, 320, L49

Iglesias, C. A., \& Rogers, F. J. 1996, ApJ, 464, 943

Izotov, Y. I., Thuan, T. X., \& Lipovetsky, V. A. 1997, ApJS, 108, 1

Kern, P., Malbet, F., Berger, J.-Ph., et al. 2002, SPIE, 4838, 76

Kervella, P., Coudé du Foresto, V., Glindemann, A., \& Hofmann, R. 2000, SPIE, 4006, 31

Kervella, P., Gitton, Ph., Ségransan, D., et al. 2002, SPIE, 4838, 858

Kervella, P., Thévenin, F., Ségransan, D., et al. 2003, A\&A, 404, 1087

Kervella, P., Ségransan, D., \& Coudé du Foresto, V. 2004a, A\&A, in press [arXiv: astro-ph0406625]

Kervella, P., Thévenin, F., Di Folco, E., \& Ségransan, D. 2004b, A\&A in press [arXiv: astro-ph0404180]

Kuchner, M. J., \& Holman, M. J. 2003, ApJ, 588, 1110

Kurucz, R. 1992, The Stellar Populations of Galaxies, IAU Symp., 149,225

Lachaume, R., Dominik, C., Lanz, T., \& Habing, H. J. 1999, A\&A, 348, 897

Lagage, P. O., \& Pantin, E. 1994, Nature, 369, 628

Lanz, T., Heap, S. R., \& Hubeny, I. 1995, ApJL, 447, 41

Laureijs, R. J., Jourdain de Muizon, M., Leech, K., et al. 2002, A\&A, 387,285

Laurent, E., Rousselet-Perraut, K., Benech, P., et al. 2002, A\&A, 390, 1171

Leinert, C., Graser, U., Waters, L. B. F. M., et al. 2000, SPIE, 4006, 43

Malfait, K., Bogaert, E., \& Waelkens, C. 1998, A\&A, 331, 211

Mihalas, D. 1978, Stellar Atmospheres, 2nd ed. (San Francisco: W. H. Freeman and Co.)

Monnier, J. D., \& Millan-Gabet, R. 2002, ApJ, 579, 694

Morel, P. 1997, A\&AS, 124, 597

Morel, P., \& Thévenin, F. 2002, A\&A, 390, 611

Mouillet, D., Larwood, J. D., Papaloizou, J. C. B., \& Lagrange, A. M. 1997, MNRAS, 292, 896

Ortega, V. G., de la Reza, R., Jilinski, E., \& Bazzanella, B. 2002, ApJ, 575, L75

Pagel, B. E. J., \& Portinari, L. 1998, MNRAS, 298, 747

Paquette, C., Pelletier, C., Fontaine, G., \& Michaud, G. 1986, ApJS, 61,177 
Pantin, E., Lagage, P. O., \& Artymowicz, P. 1997, A\&A, 327, 1123

Perryman, M. A. C., Lindegren, L., \& Kovalevsky, J. 1997, A\&A, 323, L49

Petrov, R., Malbet, F., Richichi, A., et al. 2000, Proc. SPIE, 4006, 68 Pijpers, F. P., Teixeira, T. C., Garcia, P. J., et al. 2003, A\&A, 406, L15 Quillen, A. C., \& Thorndike, S. 2002, ApJ, 578, L149

Ribas, I., Jordi, C., \& Giménez, À. 2000, MNRAS, 318, L55

Royer, F., Gerbaldi, M., Faraggiana, R., \& Gomez, A. E. 2002, A\&A, 381, 105

Saar, S. H., \& Osten, R. A. 1997, MNRAS, 284, 803

Santos, N. C., Israelian, G., \& Mayor, M. 2001, A\&A, 373, 1019

Schaller, G., Schaerer, D., Meynet, G., et al. 1992, A\&AS, 96, 269
Song, I., Caillault, J. P., \& Barrado y Navascuès, D. 2000, ApJ, 533, L41

Soubiran, C., Katz, D., \& Cayrel, R. 1998, A\&AS, 133, 221

Stapelfeldt, K. R., et al. 2004, ApJS, submitted

Thévenin, F. 1998, Chemical abundance in Late-Type Stars, CDS, Cat III/193

van Belle, G. T., Ciardi, D. R., Thompson, et al. 2001, ApJ, 559, 1155

von Zeipel, H. 1924, MNRAS, 84, 684

Wyatt, M. C., \& Dent, W. R. F. 2002, NMRAS, 334, 589

Zuckerman, B., Song, I., Bessell, M. S., \& Webb, R. A. 2001, ApJ, 562, L87 